\title{
Dynamic Optimization with Applications to Dynamic Rate Queues
}

\author{
Robert C. Hampshire \\ School of Public Policy and Management, H. John Heinz III College, Carnegie Mellon University, \\ Pittsburgh, Pennsylvania 15213, hamp@andrew.cmu.edu
}

\section{William A. Massey}

Operations Research and Financial Engineering Department, Princeton University, Princeton, New Jersey 08540, wmassey@princeton.edu

\begin{abstract}
This tutorial presents recent developments in the management of communications services and applies broadly to services involving the leasing of shared resources. These problems are more realistically modeled by queues with time-varying rates or more simply, dynamic rate queues. We first provide a review and summary of relevant results for various fundamental dynamic rate queues. The focus here is on approximations of these queueing models by low-dimensional dynamical systems.

The dynamic optimization of constrained dynamical systems is based on the calculus of variations and its various incarnations over the past three centuries. We discuss these methods in the context of Lagrangians, Hamiltonians, and Bellman value functions. Finally, we provide examples where we apply these optimization techniques to dynamic rate queues motivated by communications decision problems.
\end{abstract}

Keywords Bellman value function; calculus of variations; conservation principles; differential equations; dynamical systems; Hamiltonian; Lagrangian; Lagrange multipliers; Legendre transforms; opportunity costs; optimal control; Poisson brackets

\section{The Operations of Communication Systems and Services}

\subsection{Four Canonical Issues}

We begin by discussing the operational issues motivated by the communications industry. This is the business sector that created the telephone network in the beginning of the 20th century and gave birth to the mathematical field of queueing theory. Around the middle of the 20th century, or the 1960s, queueing theory was found to have applications to the communication of computer systems. The mathematics of queueing has taken on a life of its own since then and has applications to all services involving the leasing of shared resources.

A manager of a communications business has a continual interest in offering various services as efficiently as possible in terms of the company resources. We can express these concerns in terms of four canonical issues: performance, pricing, provisioning, and prioritization. The first one is that of service performance. These are metrics that predict the availability of resources needed for service to the typical, newly arriving customer. This is expressed in terms of the past demand and usage of these resources by previous customers.

The second issue is service pricing. If the price is too high, then no one may use the service. If the price is too low, then the cost of the resources used by the business providing the service may exceed the revenue obtained. A decision must be made as to what is the most strategic price in terms of optimizing revenue without compromising some target level of service performance. If the general strategy for a decision can be expressed in terms of a finite set of simple steps, then we call this a policy. Since the larger goal of operations 
research is to make a strategic decision, our ultimate objective is to determine an optimal policy.

The third issue is resource provisioning. This is the efficient design of the system so that just enough or an optimal number of resources are made available to achieve an acceptable target level of service performance. Here again, a policy must be developed to determine this critical amount.

Finally, there is the issue of resource prioritization. This is the efficient allocation of resources to distinct classes of customers. An optimal policy is one that accounts for the differing levels of demand, performance needs, and payment for services by these differing classes of customers.

The issue of service performance calls for managers to assume a passive role. They need only observe the history of the system to make their predictions. Classically, queueing theory has studied the static steady-state behavior of constant rate queues. We typically model these systems by time-homogeneous Markov processes. However, these models fail to capture everyday dynamic rate queueing phenomena such as the lag between the time of the peak demand for services and the time of the peak load. It is the latter time and not the former when the largest amount of service resources are needed. Hence to make more accurate performance predictions, we need to study dynamic rate queues. The underlying mathematics for these models come from the study of time-inhomogeneous Markov processes and dynamical systems.

Moreover, the remaining three issues of pricing, provisioning, and prioritization all require an active role on the part of the managers to develop optimal policies. To analyze these problems, we model the forecasted demands for these services as random counting processes. We model the resulting communication service usage as a dynamic rate queue. In turn, we model these queueing systems as discrete state Markov processes. Assuming a deterministic initial load and a finite time horizon makes the underlying Markov process transient, even if it is time homogeneous. Our goal is to develop optimal policies by applying dynamic optimization to these processes.

Finding such optimal policies can be difficult if not computationally intractable. Our approach is to develop an approximate analysis by optimizing deterministic dynamical systems that are good approximations of the given Markov process queueing models. The approximate dynamic optimization policies developed here for queueing models result in open-loop controls that depend on time and the parameters of the problem. In special cases feedback or closed-loop controls may be obtained. However, this tutorial focuses on openloop controls.

\subsection{Literature Review}

There is a vast literature in operations research relating to sequential decision making under uncertainty. We do not attempt to survey or cite all these works here. We merely point to a few streams that relate to the present tutorial and the control of dynamic rate queues.

There is a large and growing stream of research on the control of queueing systems that considers the exact optimization of steady-state queueing systems over an infinite time horizon. Sennott's book [45] examines the control of queueing systems using stochastic dynamic programming. The textbooks of Puterman [43] and Bertsekas [5], among others, provide the foundation for using Markov decision processes (MDP) for the exact optimization of stationary queueing systems. Miller [39] provides optimality conditions for finite horizon continuous time MDP with finite action spaces. There are many researchers who have extended the MDP framework to develop policies for nonhomogeneous stochastic models with infinite actions spaces. As an example of this stream, Lewis and Yoon [31] consider both pricing and admission controls for a multiserver queue with a periodic arrival rate over an infinite time horizon. They use a pointwise stationary approximation (PSA) of the queueing process (see Green and Kolesar [12]). An optimization problem is then solved over each disjoint 
time interval where stationarity is assumed. Additionally, the work of Fu et al. [11] considers the staffing of a transient queue using a pointwise stationary approximation and a nonhomogeneous MDP.

Another stream of work develops finite horizon analysis and approximations for nonhomogeneous MDP. A series of papers by Alden and Smith [2], Bean et al. [3], Hopp [21], and Hopp et al. [22] develop optimality conditions and rolling horizon methods for nonhomogeneous MDP.

We focus on the approximate dynamic optimization of dynamic rate queues over a finite time horizon. This tutorial summarizes the stream of research that uses dynamic systems to model the service system and optimal control theory to perform the optimization. An example of a paper in this stream is the work of Agnew [1], who considers dynamic control for congestion prone systems. The authors have employed the methods developed in this tutorial in a series of papers (Hampshire [14], Hampshire et al. [18, 19]).

\section{Fundamental Dynamic Rate Queues}

In this section we give a brief summary of queueing theory from a communications perspective. The first queueing paper is said to have been written in 1917 by Erlang [7] to determine the blocking probability ("all circuits are busy") for telephone trunk lines. A natural historical motivation for the field is to organize queueing models into the following four fundamental types: service demand models, revenue demand models, loss models, and delay models.

\subsection{Service Demand Models}

Service demand is analyzed through the study of arrival traffic models where we simply count the number of customers making service requests. For dynamic rates, we can start with the following simple assumptions for an arrival process. We define a process $\{A(t) \mid t \geq 0\}$ in terms of a positive, deterministic function $\{\lambda(t) \mid t \geq 0\}$, where

(1) We have a simple (unit jump) random counting process $\{A(t) \mid t \geq 0\}$.

(2) The mean number of jumps in a time interval $(s, t]$ is $\int_{s}^{t} \lambda(r) d r$.

(3) The random numbers of jumps during disjoint times intervals are mutually independent random variables.

By a theorem due to Prékopa [42], these assumptions uniquely characterize a nonhomogeneous Poisson process, where

$$
P\{A(t)-A(s)=n\}=\exp \left(-\int_{s}^{t} \lambda(r) d r\right) \frac{\left(\int_{s}^{t} \lambda(r) d r\right)^{n}}{n !}
$$

for all integers $n \geq 0$. If we view customers as a large population of independent actors, then these three assumptions are reasonable modeling assumptions. This makes the Poisson process a natural model for service demand traffic. The only adjustment that needs to be made from the perspective of classical queueing theory is to consider nonhomogeneous as well as homogeneous Poisson processes.

\subsection{Service Resource Demand Models}

Service resource demand is the total amount of service resources that are requested by the customers at any given time. Communication examples of such resources are telephone trunk lines, radio channels, bandwidth, or call center agents. The simplest service resource demand queueing models are ones with nonhomogeneous Poisson arrivals and an infinite number of servers where the service time is the service resource. Since the resources that customers request are not necessarily the same as the resources they actually receive, we 
can model these requests as a sequence of independent and identically distributed, positive random variables. In classical telephony, this type of service demand is referred to as the offered load.

For the resulting offered load process $\left\{Q_{\infty}(t) \mid t \geq 0\right\}$, each $Q_{\infty}(t)$ has a Poisson distribution or

$$
P\left\{Q_{\infty}(t)=n\right\}=e^{-q_{\infty}(t)} \cdot \frac{q_{\infty}(t)^{n}}{n !}
$$

for all positive integers $n$, including zero. However, this offered load process is not a Poisson process. As discussed in Eick et al. [6], the resulting deterministic, mean offered load process $\left\{q_{\infty}(t) \mid t \geq 0\right\}$ equals

$$
q_{\infty}(t) \equiv E\left[\int_{t-S}^{t} \lambda(r) d r\right]=E\left[\lambda\left(t-S_{e}\right)\right] \cdot E[S],
$$

where the random variable $S$ has the same distribution as any of the i.i.d. service times. The random variable $S_{e}$ is defined to have a stationary excess distribution from $S$, where

$$
P\left\{S_{e} \leq t\right\} \equiv \frac{1}{E[S]} \int_{0}^{t} P\{S>s\} d s .
$$

This is the distribution for the remaining time of an interrupted service from a renewal process for these i.i.d. times. If $S$ has a finite second moment, then $S_{e}$ has a finite mean where

$$
E\left[S_{e}\right]=\frac{E\left[S^{2}\right]}{2 \cdot E[S]}
$$

It can be shown that the distributions for $S$ and $S_{e}$ are the same if and only if $S$ is exponentially distributed or

$$
S_{e} \stackrel{\mathrm{d}}{=} S \text { if and only if } P\{S>t\}=e^{-\mu t} \quad \text { where } E[S]=1 / \mu
$$

From the properties of the Poisson distribution, it follows that $E\left[Q_{\infty}(t)\right]=q_{\infty}(t)$ for all $t \geq 0$. We can make this offered load process Markov by letting the service times (the amount of time customers are in service) have an exponential distribution with mean rate $\mu$. The mean offered load $\left\{q_{\infty}(t) \mid t \geq 0\right\}$ then solves the ordinary differential equation

$$
\frac{d}{d t} q_{\infty}(t)=\lambda(t)-\mu \cdot q_{\infty}(t)
$$

Observe that for the case of $\lambda$ being a constant, we have

$$
\lim _{t \rightarrow \infty} q_{\infty}(t)=\rho \equiv \lambda / \mu \text {. }
$$

For the time-homogeneous case, $\rho$ equals the steady-state mean offered load.

\subsection{Service Loss Models}

Inspired by voice communications, service loss models are one way to handle the reality of limited resources. Customers who arrive when there are no available service resources are told to go away. In telephony, this is the experience of receiving the message that all circuits are busy. For the case of a constant rate or time-homogeneous Poisson arrival process and a finite number of $C$ servers, we have the classical Erlang loss model or $M / M / C / C$ queue. The long run steady-state probability of all circuits being busy is given by $\beta_{C}(\rho)$, defined as

$$
\beta_{C}(\rho) \equiv \frac{\rho^{C}}{C !} / \sum_{i=0}^{C} \frac{\rho^{i}}{i !},
$$

where $\rho$ is the steady-state mean offered. 
Using the theory of time-reversible Markov processes (see Kelly [27]), we can also show that

$$
\beta_{C}(\rho)=\lim _{t \rightarrow \infty} P\left\{Q_{C / C}(t)=C\right\}=\lim _{t \rightarrow \infty} P\left\{Q_{\infty}(t)=C \mid Q_{\infty}(t) \leq C\right\} .
$$

In general, the steady-state distribution for the Erlang loss system is the steady-state distribution for the offered load system conditioned on being less than or equal to the number of servers $C$. Because of the insensitivity results for the Erlang loss model in steady state, these steady-state distributional results extend to the non-Markovian case of general service distributions.

For the case of nonhomogeneous Poisson arrivals, however, the transient probability of blocking for the Erlang loss model no longer has this conditional Poisson distribution. This is true despite the fact that its offered load counterpart still has a Poisson distribution. Regardless, this conditional Poisson distribution is still a good estimation of the transient distribution for the Erlang loss model and this technique is called the modified offered load approximation (see Jagerman [24] as well as Massey and Whitt [37]).

We can still use the dynamical system of the mean offered load for approximating the performance of the loss system queueing model, if we constrain the dynamical system to stay below some given threshold value. In practice, given $C$ and $0<\epsilon<1$, we need to find the mean offered load value $\theta$, where

$$
\beta_{C}(\theta)=\epsilon
$$

The modified offered load approximation then tells us that the dynamics of the event,

$$
\max _{0 \leq t \leq T} P\left\{Q_{C / C}(t)=C\right\} \leq \epsilon,
$$

can be estimated by the approximating event of

$$
\max _{0 \leq t \leq T} q_{\infty}(t) \leq \theta
$$

\subsection{Service Delay Models}

Service delay models, as inspired by data communications, are another way to handle the allocation of scarce resources. The customers that arrive when the necessary resources are not available for service are told to wait until the resources are available. An example is the delay experienced when downloading a file from a Web server that is heavily used. Kleinrock [28] showed that a single server queue with a processor sharing (PS) service discipline could approximate the performance of CPU server with a round robin service discipline. Now let the constant $\lambda$ be the Poisson arrival rate and the constant $1 / \mu$ be the average size for an exponentially distributed job size for the queueing process $\left\{Q_{1 / \infty}^{\mathrm{PS}}(t) \mid t \geq 0\right\}$. Using the theory for steady-state distributions of Markov chains and processes gives us

$$
\lim _{t \rightarrow \infty} P\left\{Q_{1 / \infty}^{\mathrm{PS}}(t)=n\right\}= \begin{cases}(1-\rho) \rho^{n} & \text { if } \rho<1 \\ 0 & \text { if } \rho \geq 1\end{cases}
$$

The PS service discipline has a natural scale invariance that leaves service times unchanged. If we set $\eta$ equal to a positive integer, then dividing each job into $\eta$ even parts and processing these jobs in parallel does not change the processing time of the collective original job. The $\eta$-fold i.i.d. superposition of nonhomogeneous Poisson arrivals with rate function $\lambda(t)$ yields a nonhomogeneous Poisson process with rate function $\eta \cdot \lambda(t)$. Jobs whose size is exponentially distributed with rate $\mu$ are then exponentially distributed with rate $\eta \cdot \mu$ when divided by the scale factor $\eta$. This is a motivation for the asymptotic analysis 
of uniform acceleration for time-inhomogeneous Markov processes (see Massey [35] and later Massey and Whitt [38]).

If we let $\left\{Q_{1 / \infty}^{\mathrm{PS}}(t ; \eta) \mid t \geq 0\right\}$ be this newly scaled queueing process, then we have

$$
\lim _{\eta \rightarrow \infty} P\left\{Q_{1 / \infty}^{\mathrm{PS}}(t ; \eta)=n\right\}= \begin{cases}(1-\rho(t)) \rho(t)^{n} & \text { if } \rho_{*}(t)<1, \\ 0 & \text { if } \rho_{*}(t) \geq 1,\end{cases}
$$

where

$$
\rho(t) \equiv \lambda(t) / \mu \quad \text { and } \quad \rho_{*}(t) \equiv \sup _{0 \leq s<t} \frac{\int_{s}^{t} \lambda(r) d r}{\mu \cdot(t-s)} .
$$

A proof of this result can be found in both Massey [35, 36]. The PS interpretation of uniform acceleration is discussed in Hampshire et al. [17].

Through the theory of strong approximations (see Kolmós et al. [29], Ethier and Kurtz [8]), we can also apply these same uniform acceleration asymptotics directly to the random sample path behavior of this queueing process and obtain

$$
\lim _{\eta \rightarrow \infty} \sup _{0 \leq t \leq T}\left|\frac{1}{\eta} Q_{1 / \infty}^{\mathrm{PS}}(t ; \eta)-q_{1 / \infty}^{\mathrm{PS}}(t)\right|=0 \quad \text { a.s. }
$$

where if $q_{1 / \infty}^{\mathrm{PS}}(0)=0$, then

$$
q_{1 / \infty}^{\mathrm{PS}}(t) \equiv \max _{0 \leq t \leq T} \int_{t}^{T}(\lambda(s)-\mu) d s
$$

The resulting deterministic process $\left\{q_{1 / \infty}^{\mathrm{PS}}(t) \mid t \geq 0\right\}$ is called the fluid approximation for single server queues as discussed in Kleinrock [28]. The proof of this functional strong law of large numbers is given in Mandelbaum and Massey [32]. Note that the fluid model is also a nonsmooth dynamical system of the form

$$
\frac{d}{d t} q_{1 / \infty}^{\mathrm{PS}}(t)=(\lambda(t)-\mu) \cdot\left\{q_{1 / \infty}^{\mathrm{PS}}(t)>0\right\}+(\lambda(t)-\mu)^{+} \cdot\left\{q_{1 / \infty}^{\mathrm{PS}}(t)=0\right\},
$$

where $q_{1 / \infty}^{\mathrm{PS}}(0)=0$. The term $\left\{q_{1 / \infty}^{\mathrm{PS}}(t)>0\right\}$ is a binary valued indicator function that has the value one if $q_{1 / \infty}^{\mathrm{PS}}(t)>0$ is true and zero otherwise.

Another simple example of delay is in a telephone call where customers are hearing music instead of a message that all circuits are busy. This can be modeled by a multiserver queue with nonhomogeneous Poisson arrivals, an infinite capacity waiting space, and a first-come, first-served (FCFS) customer service discipline. The number of servers maps $C$ to the number of telephone call center agents. From a managerial perspective, a natural asymptotic analysis is to scale up the average rate of arriving customers from $\lambda$ to $\eta \cdot \lambda$, where $\eta>1$, and scale the number of servers from $C$ to $\eta \cdot C$. We are simultaneously scaling up the "supply" of service resources and the "demand" of the customer arrival rate. Such a scaling, in the language of call centers, is called resource pooling. It was first considered asymptotically by Halfin and Whitt [13], and this type of scaling is defined to be in the quality efficiency driven (QED) regime according to Zeltyn and Mandelbaum [47]. Dividing out this new Markov process by this same scaling parameter $\eta$ makes the resulting ratio converge to a deterministic dynamical system $\left\{q_{C / \infty}^{\mathrm{FCFS}}(t) \mid t \geq 0\right\}$, where

$$
\lim _{\eta \rightarrow \infty} \sup _{0 \leq t \leq T}\left|\frac{1}{\eta} Q_{C / \infty}^{\mathrm{FCFS}}(t ; \eta)-q_{C / \infty}^{\mathrm{FCFS}}(t)\right|=0 \quad \text { a.s. }
$$


with

$$
\frac{d}{d t} q_{C / \infty}^{\mathrm{FCFS}}(t)=\lambda(t)-\mu \cdot \min \left(q_{C / \infty}^{\mathrm{FCFS}}(t), C\right)
$$

and $q_{C / \infty}^{\mathrm{FCFS}}(0) \equiv Q_{C / \infty}^{\mathrm{FCFS}}(0)$. This new variant or $\mathrm{QED}$ version of uniform acceleration applies to a large class of Markov processes that we call Markovian service networks. As was shown in Mandelbaum et al. [33], all networks of this type have a strong law of large numbers limit theorem when uniformly accelerated. Moreover, the limiting deterministic process is always a dynamical system.

Finally, we revisit the single server with the PS service discipline. This time we add a permanent job that interacts with all the other jobs being processed. We can use this process to model the response time for a tagged customer entering the system at some specific time and denote this service discipline by PS+. As shown in Hampshire and Massey [16], this system also fits into the framework of being a Markovian service network. The result is almost sure convergence, uniformly on compact intervals of time, or

$$
\lim _{\eta \rightarrow \infty} \sup _{0 \leq t \leq T}\left|\frac{1}{\eta} Q_{1 / \infty}^{\mathrm{PS}+}(t ; \eta)-q_{1 / \infty}^{\mathrm{PS}+}(t)\right|=0 \quad \text { a.s. }
$$

to the deterministic dynamical system $\left\{q_{1 / \infty}^{\mathrm{PS}+}(t) \mid t \geq 0\right\}$, which is uniquely characterized by its initial value and the ordinary differential equation

$$
\frac{d}{d t} q_{1 / \infty}^{\mathrm{PS}+}(t)=\lambda(t)-\frac{\mu \cdot q_{1 / \infty}^{\mathrm{PS}+}(t)}{1+q_{1 / \infty}^{\mathrm{PS}+}(t)}
$$

\subsection{Resource Demand Dynamics}

A recurring theme in dynamic rate queues is that the time of the peak arrival rate is not the time of the peak mean offered load. The timing of the latter always has a positive lag after the timing of the former. A similar lag occurs for the times of the minimum arrival rate and the minimal mean offered load. We can illustrate through an example this phenomena with the dynamical system for the mean offered load.

We can visualize the time evolution of this mean offered load $\left\{q_{\infty}(t) \mid t \geq 0\right\}$ by viewing it as a one-dimensional dynamical system. In Figure 1, we make a phase-space plot of the time derivative for $q_{\infty}(t)$ versus its own time derivative for all time $t \geq 0$. For constant $\lambda$, we have a continuous evolution of the dynamical system along the phase-space equation of a line, according to the ordinary differential equation. This line has $\lambda$ as its $y$-intercept and $-\mu$ as its slope. When the time derivative of $q_{\infty}(t)$ is positive, then the flow is toward the right or is increasing over time along the $x$-axis, when the line for the dynamical system flow is above the $x$-axis. Conversely, the system flow is from right to left when the flow line is below the $x$-axis. The phase-space plot reveals that all solutions to the differential equation (i.e., regardless of the initial value $\left.q_{\infty}(0)\right)$ converge to $\rho=\lambda / \mu$ as $t$ goes to infinity.

When $\lambda$ is time dependent, then the time evolution of the mean offered load process is no longer along a straight line. Moreover, the limit of $q_{\infty}(t)$ as $t$ approaches infinity may no longer converge to a unique point in phase space. Suppose that we let the arrival rate of $\lambda$ be periodic, we can show that the limiting behavior of $q_{\infty}$ is also periodic over time. More informally, if the period is a "day," then we have "time of day" limits for what we call the periodic steady-state distribution.

Now consider the following example from Horne et al. [23]. When we set $\lambda(t)=a+\hat{a} \cdot \sin (b t)$ with $b>0$, then the periodic steady-state behavior of the mean offered load phase-space process moves in a clockwise elliptical orbit as shown in Figure 2. The center of this closed curve is at $(a / \mu, 0)$. Moreover, the ellipse has both foci on the $x$-axis with extremal radii equal to $\hat{a} / \sqrt{b^{2}+\mu^{2}}$ and $\hat{a} b / \sqrt{b^{2}+\mu^{2}}$. If we draw a line of slope $-\mu$ through any point on 
Figure 1. Phase-space plot of the mean offered load dynamical system with a constant arrival rate.

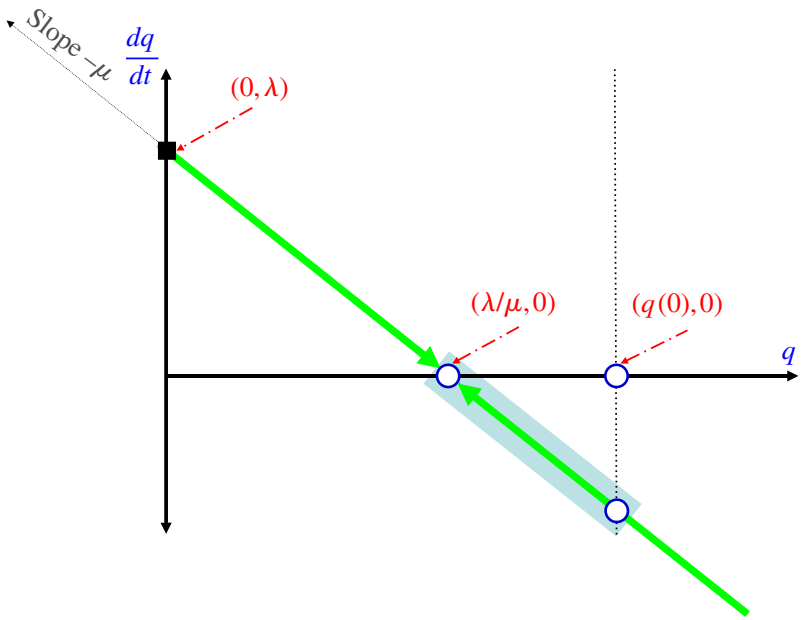

the phase-space curve, then the $y$-intercept of the line gives the value of the arrival rate $\lambda$ at the time of the phase-space point.

Now we show how our service resource demand model can help us manage a resource provisioning problem for our service loss model. First, we define

$$
C_{*}(\epsilon, \rho) \equiv \min \left\{C \mid \beta_{C}(\rho) \leq \epsilon\right\} .
$$

The integer value $C_{*}(\epsilon, \rho)$ equals the smallest number of servers needed such that the Erlang blocking formula is less than or equal to $\epsilon$ for the given steady-state mean offered load of $\rho$. The modified offered load approximation says that the scheduling policy of $\{C(t) \mid 0 \leq t \leq T\}$, where

$$
C(t) \equiv C_{*}\left(\epsilon, q_{\infty}(t)\right)
$$

is a good estimate of the smallest number of servers needed to keep the blocking probability below $\epsilon$ over the interval $(0, T]$. Hampshire et al. [20] develop this approach in the context of bandwidth trading.

FIgURE 2. Phase-space plot of the mean offered load dynamical system with a sinusoidal arrival rate.

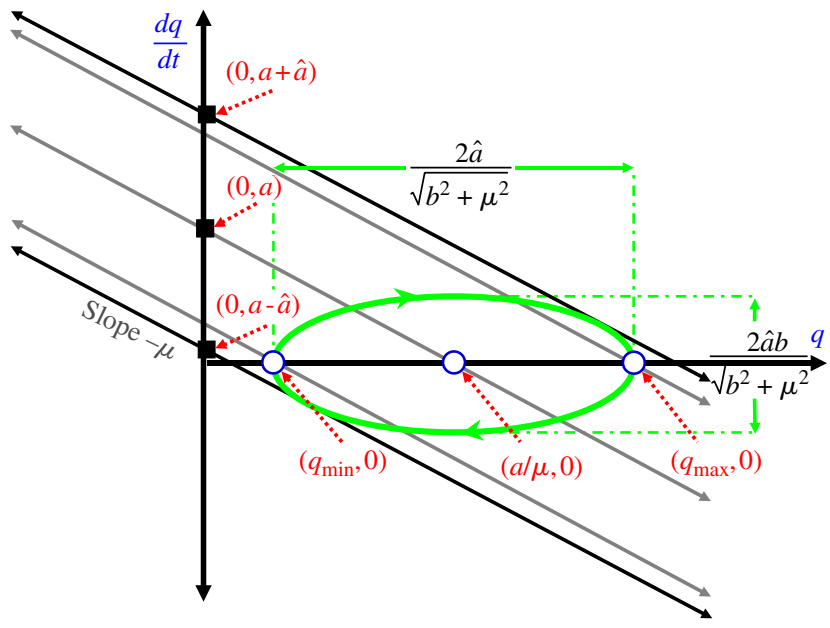




\subsection{Fundamental Decision Problems}

We now introduce specific types of decision problems for dynamic rate queues that are motivated by more than just optimizing the performance of the queueing model. Suppose that we want to develop a dynamic pricing policy $\{\pi(t) \mid 0 \leq t \leq T\}$ for our service loss model, where the traffic arrival rate is now a given function of time $t$ and the price $\pi(t)$; i.e., $\lambda=\lambda(t, \pi(t))$. Our goal is to maximize the average revenue given the constraint that the probability of blocking never exceeds some predetermined quality of service level $\epsilon$. Thus we want to determine a policy that gives the maximum expected revenue value $R(T)$, where

$$
R(T) \equiv \max _{\{\pi(t) \mid 0 \leq t \leq T\}} \int_{0}^{T} \pi(t) \lambda(t, \pi(t)) \cdot P\left\{Q_{C / C}(t)<C\right\} d t .
$$

In $§ 9.1$, we discuss how to use this calculus of variations machinery to find an approximate optimal policy for this pricing problem. We do so by finding the optimal policy for an associated dynamical system or fluid model.

Now consider a multiserver, service delay model where the revenue received from each customer is $r$ and the total wages (cost rate) for the $C$ servers equals $w(C)$. A natural goal is to find a server staffing policy $\{C(t) \mid 0 \leq t \leq T\}$ that maximizes the average profit. We now want to determine a policy that gives us some expected revenue value $R(T)$, where

$$
R(T) \equiv \max _{\{C(t) \mid 0 \leq t \leq T\}} \int_{0}^{T} r \cdot E\left[Q_{C(t) / \infty}^{\mathrm{FCFS}}(t)\right]-w(C(t)) d t
$$

In $\S 9.2$, we discuss how we can use the calculus of variations machinery to find an approximate optimal policy for this provisioning problem. We do so by finding the exact optimal policy for an associated dynamical system or fluid model.

Finally, consider a single server, two customer class, service delay model, with a weighted PS discipline and a permanent customer. The revenue received from each class is $r_{i}$ per customer for $i=1,2$. A natural objective is to find the processing weights for each class that maximizes the average profit. We now want to determine a policy that gives us some expected revenue value $R(T)$, where

$$
R(T) \equiv \max _{\{w(t) \mid 0 \leq t \leq T\}} \int_{0}^{T} \mathbf{r} \cdot E\left[\boldsymbol{\theta}\left(\mathbf{w}, \mathbf{Q}_{1 / \infty}^{W} \mathrm{PS}+(t)\right)\right] d t .
$$

In $\S 9.3$, we discuss how to use this calculus of variations machinery to find an approximate optimal policy for this prioritization problem. We do so by finding the exact optimal policy for an associated dynamical system or fluid model.

\section{Three Centuries of Dynamic Optimization}

Classical mechanics was developed in the 18th and 19th centuries as an elegant mathematical summary for the 17th-century physics of Newtonian mechanics. The work of Lagrange and Hamilton forms the basis for these techniques. The mathematical techniques for dynamic optimization evolved during the 20th century in the field of operations research. This effort was led by Richard Bellman, who unified both the Lagrangian and Hamiltonian approaches while developing a comprehensive framework for optimal control and decision analysis.

The 18th-century work of Lagrange describes the optimal dynamics or trajectories of physical mechanical systems. Position and velocity are the fundamental quantities used to describe the state of the system. The key notions of Lagrange's approach, constructed as a function of the position, velocity, and time, center around the concepts of the action integral and its integrand, the Lagrangian. Despite the fact that action does not have a meaningful, classical mechanical interpretation, Lagrange's use of the quantity led to the same dynamics 
as Newtonian mechanics. Lagrange's equations of motion are called the Euler-Lagrange equations (see Lanczos [30], Ewing [9]).

In the context of communications service management, action has the interpretation of the optimal total profit or value obtained over a finite time interval. The Lagrangian, being the integrand of the action integral, corresponds to the optimal profit rate.

In the 19th century, Hamilton reformulated many of Lagrange's results. When describing the state of the system, Hamilton continued to use position while replacing velocity by the notion of momentum. Using position and momentum, Hamilton constructed a description of the total energy of the system now known as the Hamiltonian. The coupled dynamical system, the system's optimal position and momentum variables, are known as Hamilton's equations.

If the Lagrangian of the system is not an explicit function of time, then it has a Hamiltonian that is constant over time along the optimal path. This result is known as the conservation of energy. In general, the Hamiltonian of a system is obtained by applying the Legendre transform to the Lagrangian of the same system. If the Lagrangian of the system is not an explicit function of position, then the momentum variable of the Hamiltonian is constant over time. This result is known as the conservation of momentum.

Dynamical equations can be easily solved numerically if the initial conditions are given. Unfortunately for Hamilton's equations, we are given initial value of the position variable at time zero but only the terminal value of the momentum variable at time $T$.

For our communications service operational problems, Pontryagin's principle (see Pontryagin et al. [41], Kamien and Schwartz [26], or Sethi and Thompson [46]) is equivalent to selecting a control that minimizes the Hamiltonian at every point in time if we are maximizing the profit functional.

In the 20th century, Richard Bellman unified both the Lagrangian and Hamiltonian approaches in the context of operations research while developing a comprehensive framework for decision analysis. The fundamental object of analysis for sequential decision making is the Bellman value function, or value function for short. This is the amount of profit or value obtained over a time interval assuming that the optimal decision or control is selected over that entire interval. The value function satisfies a recursive relationship known as Bellman's principle of optimality. This principle leads to a partial differential equation for the value function known as Bellman's equation (see Bellman [4]). All of the key results of classical mechanics, Lagrangian and Hamiltonian, can be obtained within the Bellman framework.

In the context of communications service management, the Bellman formulation yields the tools necessary to interpret the physical notion of momentum as the opportunity cost per customer. Furthermore, the Bellman formulation allows us to interpret the Hamiltonian or physical notion of energy as the opportunity cost rate.

In the remainder of this tutorial, we derive the classical results of Lagrange, Hamilton, and Bellman and apply them to the optimization of dynamic rate queues.

\section{The Lagrangian}

The development of dynamic optimization begins with the Lagrangian. We define it to be a twice differentiable function $\mathcal{L}: \mathbb{R}^{2} \times[0, T] \rightarrow \mathbb{R}$. We then apply this function to a differentiable path $q:[0, T] \rightarrow \mathbb{R}$ as $\mathcal{L}(q(t), \dot{q}(t), t)$, where

$$
\dot{q}(t) \equiv \frac{d}{d t} q(t) .
$$

Problem 1 (Minimizing Arc Length). Find the differentiable paths that minimize arc length over space-time, where

$$
\mathcal{L}(q(t), \dot{q}(t), t)=\sqrt{1+\dot{q}(t)^{2}}
$$


for the case of both endpoints at time 0 and $T$ being fixed and the case of just the initial point at time zero being fixed.

Solution. Using Euclidean geometry, we see that optimizing over differentiable paths with $q(0)=x$ and $q(T)=y$ yields

$$
\sqrt{T^{2}+(x-y)^{2}}=\min _{q: q(0)=x, q(T)=y}\left[\int_{0}^{T} \sqrt{1+\dot{q}(t)^{2}} d t\right],
$$

where the optimizing path is

$$
q_{*}(t)=x \cdot(1-t / T)+y \cdot t / T \quad \text { and } \quad \dot{q}_{*}(t)=\frac{y-x}{T}
$$

for all $0 \leq t \leq T$. However, if only the initial endpoint $q(0)=x$ is fixed, then we have

$$
T=\min _{q: q(0)=x}\left[\int_{0}^{T} \sqrt{1+\dot{q}(t)^{2}} d t\right],
$$

where the optimizing path is

$$
q_{*}(t)=x \quad \text { and } \quad \dot{q}_{*}(t)=0
$$

for all $0 \leq t \leq T$.

\subsection{Euler-Lagrange Equations}

Now we present the first fundamental result for the calculus of variations.

Theorem 1 (Euler-Lagrange Equations). Given an optimal path $q_{*}:[0, T] \rightarrow \mathbb{R}$ with $q_{*}(0)=x$ such that

$$
\int_{0}^{T} \mathcal{L}\left(q_{*}, \dot{q}_{*}\right)(t) d t=\underset{q: q(0)=x}{\operatorname{opt}}\left[\int_{0}^{T} \mathcal{L}(q, \dot{q})(t) d t\right]
$$

then

$$
\frac{d}{d t} \frac{\partial \mathcal{L}}{\partial \dot{q}}\left(q_{*}, \dot{q}_{*}\right)(t)=\frac{\partial \mathcal{L}}{\partial q}\left(q_{*}, \dot{q}_{*}\right)(t)
$$

with the transversality condition

$$
\frac{\partial \mathcal{L}}{\partial \dot{q}}\left(q_{*}, \dot{q}_{*}\right)(T)=0 .
$$

Proof. If $\phi:[0, T] \rightarrow \mathbb{R}$ is differentiable with $\phi(0)=0$, then $q_{*}+\epsilon \cdot \phi$ for any constant $\epsilon$ is a differentiable path that starts at $x$. This means that

$$
\mathcal{L}\left(q_{*}, \dot{q}_{*}\right) \succeq_{\text {opt }} \mathcal{L}\left(q_{*}+\epsilon \phi, \dot{q}_{*}+\epsilon \dot{\phi}\right),
$$

for all $\epsilon$. Since the optimum corresponds to the case of $\epsilon=0$, the total derivative, with respect to $\epsilon$, of the integral of this Lagrangian over the interval $[0, T]$ must be zero when $\epsilon=0$. Using integration by parts and the fact that $\phi(0)=0$ yields

$$
\begin{aligned}
0 & =\left.\frac{d}{d \epsilon}\right|_{\epsilon=0} \int_{0}^{T} \mathcal{L}\left(q_{*}+\epsilon \cdot \phi, \dot{q}_{*}+\epsilon \cdot \dot{\phi}\right)(t) d t \\
& =\int_{0}^{T}\left(\frac{\partial \mathcal{L}}{\partial q}\left(q_{*}, \dot{q}_{*}\right)(t) \cdot \phi(t)+\frac{\partial \mathcal{L}}{\partial \dot{q}}\left(q_{*}, \dot{q}_{*}\right)(t) \cdot \dot{\phi}(t)\right) d t \\
& =\frac{\partial \mathcal{L}}{\partial \dot{q}}\left(q_{*}, \dot{q}_{*}\right)(T) \cdot \phi(T)+\int_{0}^{T}\left(\frac{\partial \mathcal{L}}{\partial q}-\frac{d}{d t} \frac{\partial \mathcal{L}}{\partial \dot{q}}\right)\left(q_{*}, \dot{q}_{*}\right)(t) \cdot \phi(t) d t .
\end{aligned}
$$

If we first assume that $\phi(T)=0$, then the remaining integrand can only be zero if the Euler-Lagrange equations are satisfied. We then return to the case of $\phi(T) \neq 0$ to obtain the transversality condition. 
Example 1 (Newtonian Mechanics). Define the Lagrangian of a particle to be

$$
\mathcal{L}(q, \dot{q}) \equiv \frac{1}{2} m \cdot(\dot{q})^{2}-V(q),
$$

where $V(q)$ is the potential energy of a particle and the first term is the kinetic energy. The Euler-Lagrange equations for this Lagrangian are

$$
(m \dot{q})^{\cdot}=-V^{\prime}(q),
$$

with the transversal condition of $m(T) \cdot \dot{q}(T)=0$. When the mass $m$ is constant, then this equation reduces to the Newtonian law of "force equals mass times acceleration," where this (conservative) force is the derivative (gradient) of a potential.

\subsection{Terminal Values and Sensitivity Analysis}

Corollary 1 (Lagrangian with a Terminal Value Function). Now we are also given a terminal value function $l: \mathbb{R} \rightarrow \mathbb{R}^{2}$ that is differentiable. If we have an optimal path $q_{*}:[0, T] \rightarrow \mathbb{R}$, where

$$
\int_{0}^{T} \mathcal{L}\left(q_{*}, \dot{q}_{*}\right)(t) d t+l\left(q_{*}\right)(T)=\underset{q: q(0)=x}{\operatorname{opt}}\left[\int_{0}^{T} \mathcal{L}(q, \dot{q})(t) d t+l\left(q_{*}\right)(T)\right],
$$

then $q_{*}$ solves the same Euler-Lagrange equations as given by Equation (1). However, the transversality condition is now

$$
\frac{\partial \mathcal{L}}{\partial \dot{q}}\left(q_{*}, \dot{q}_{*}\right)(T)+\frac{\partial l}{\partial q}\left(q_{*}\right)(T)=0 .
$$

Proof. Using the total time derivative, we can rewrite the terminal value as

$$
\begin{aligned}
l(q)(T) & =l(q)(0)+\int_{0}^{T} \frac{d}{d t} l(q)(t) d t \\
& =l(q)(0)+\int_{0}^{T} \frac{\partial l}{\partial q}(q)(t) \cdot \dot{q}(t)+\frac{\partial l}{\partial t}(q)(t) d t .
\end{aligned}
$$

This means that we can transform our optimization problem into one without a terminal value function, where the Lagrangian $\mathcal{L}$ is now

$$
\hat{\mathcal{L}}(q, \dot{q})(t)=\mathcal{L}(q, \dot{q})(t)+\frac{\partial l}{\partial q}(q)(t) \cdot \dot{q}(t)+\frac{\partial l}{\partial t}(q)(t) .
$$

Finally, we observe that $\hat{\mathcal{L}}$ generates the same Euler-Lagrange equations as $\mathcal{L}$ but yields different transversality conditions.

Corollary 2 (Sensitivity Analysis). If $q_{*}:[0, T] \rightarrow \mathbb{R}$ is an optimal path, where

$$
\int_{0}^{T} \mathcal{L}\left(q_{*}, \dot{q}_{*}\right)(t) d t=\underset{q: q(0)=x}{\operatorname{opt}}\left[\int_{0}^{T} \mathcal{L}(q, \dot{q})(t) d t\right],
$$

then for any time-constant parameter a, we have

$$
\frac{d}{d a} \int_{0}^{T} \mathcal{L}\left(q_{*}, \dot{q}_{*}\right)(t) d t=-\frac{\partial \mathcal{L}}{\partial \dot{q}}\left(q_{*}, \dot{q}_{*}\right)(0) \cdot \frac{d q}{d a}(0)+\int_{0}^{T} \frac{\partial \mathcal{L}}{\partial a}\left(q_{*}, \dot{q}_{*}\right)(t) d t
$$


Proof. Using Theorem 1 yields

$$
\begin{array}{rl}
\frac{d}{d a} \int_{0}^{T} & \mathcal{L}\left(q_{*}, \dot{q}_{*}\right)(t) d t \\
= & \int_{0}^{T}\left(\frac{\partial \mathcal{L}}{\partial q}\left(q_{*}, \dot{q}_{*}\right)(t) \cdot \frac{d q}{d a}(t)+\frac{\partial \mathcal{L}}{\partial \dot{q}}\left(q_{*}, \dot{q}_{*}\right)(t) \cdot \frac{d \dot{q}}{d a}(t)+\frac{\partial \mathcal{L}}{\partial a}\left(q_{*}, \dot{q}_{*}\right)(t)\right) d t \\
= & \frac{\partial \mathcal{L}}{\partial \dot{q}}\left(q_{*}, \dot{q}_{*}\right)(T) \cdot \frac{d q}{d a}(T)-\frac{\partial \mathcal{L}}{\partial \dot{q}}\left(q_{*}, \dot{q}_{*}\right)(0) \cdot \frac{d q}{d a}(0)+\int_{0}^{T} \frac{\partial \mathcal{L}}{\partial a}\left(q_{*}, \dot{q}_{*}\right)(t) d t \\
& +\int_{0}^{T}\left(\frac{\partial \mathcal{L}}{\partial q}-\frac{d}{d t} \frac{\partial \mathcal{L}}{\partial \dot{q}}\right)\left(q_{*}, \dot{q}_{*}\right)(t) \cdot \frac{d q}{d a}(t) d t \\
= & \int_{0}^{T} \frac{\partial \mathcal{L}}{\partial a}\left(q_{*}, \dot{q}_{*}\right)(t) d t .
\end{array}
$$

\section{Constrained Lagrangian Dynamics}

\subsection{Multipliers and Slack Variables}

We can model the optimization of a Lagrangian subjected to equality constraints as follows.

Theorem 2 (Equality Constraints). If $\Phi: \mathbb{R}^{2} \rightarrow \mathbb{R}$ is a differentiable function, then

$$
\underset{\substack{q: q(t)=x \\ \Phi(q, \dot{q})=0}}{\operatorname{opt}}\left[\int_{0}^{T} \mathcal{L}(q, \dot{q})(t) d t\right]=\underset{q: q(t)=x}{\operatorname{opt}}\left[\int_{0}^{T} \mathcal{L}(q, \dot{q})(t)+r(t) \cdot \Phi(q, \dot{q})(t) d t\right] .
$$

Moreover, the optimizing paths $q_{*}:[0, T] \rightarrow \mathbb{R}$ and $r_{*}:[0, T] \rightarrow \mathbb{R}$ satisfy the Euler-Lagrange equations

$$
\frac{d}{d t}\left(\frac{\partial \mathcal{L}}{\partial \dot{q}}\left(q_{*}, \dot{q}_{*}\right)+r_{*} \cdot \frac{\partial \Phi}{\partial \dot{q}}\left(q_{*}, \dot{q}_{*}\right)\right)(t)=\left(\frac{\partial \mathcal{L}}{\partial q}\left(q_{*}, \dot{q}_{*}\right)+r_{*} \cdot \frac{\partial \Phi}{\partial q}\left(q_{*}, \dot{q}_{*}\right)\right)(t),
$$

with the equality constraint

$$
\Phi\left(q_{*}, \dot{q}_{*}\right)(t)=0
$$

and the transversality condition

$$
\left(\frac{\partial \mathcal{L}}{\partial \dot{q}}\left(q_{*}, \dot{q}_{*}\right)+r_{*} \cdot \frac{\partial \Phi}{\partial \dot{q}}\left(q_{*}, \dot{q}_{*}\right)\right)(T)=0 .
$$

Proof. Define the Lagrangian $\hat{\mathcal{L}}$, where

$$
\hat{\mathcal{L}}(q, \dot{q}, r) \equiv \mathcal{L}(q, \dot{q})+r \cdot \Phi(q, \dot{q}),
$$

and we are done.

We now model the optimization of a Lagrangian subjected to inequality constraints.

Theorem 3 (Inequality Constraints). If $\Psi: \mathbb{R}^{2} \rightarrow \mathbb{R}$ is a differentiable mapping, then

$$
\underset{\substack{q: q(t)=x \\ \Psi(q, \dot{q}) \geq 0}}{\operatorname{opt}}\left[\int_{0}^{T} \mathcal{L}(q, \dot{q})(t) d t\right]=\underset{q: q(t)=x}{\operatorname{opt}}\left[\int_{0}^{T} \mathcal{L}(q, \dot{q})(t)+r(t) \cdot\left(\Psi(q, \dot{q})(t)-\sigma(t)^{2}\right) d t\right] .
$$


Moreover, the optimizing paths $q_{*}:[0, T] \rightarrow \mathbb{R}$ and $r_{*}:[0, T] \rightarrow \mathbb{R}$ satisfy the Euler-Lagrange equations

$$
\frac{d}{d t}\left(\frac{\partial \mathcal{L}}{\partial \dot{q}}\left(q_{*}, \dot{q}_{*}\right)+r_{*} \cdot \frac{\partial \Psi}{\partial \dot{q}}\left(q_{*}, \dot{q}_{*}\right)\right)(t)=\left(\frac{\partial \mathcal{L}}{\partial q}\left(q_{*}, \dot{q}_{*}\right)+r_{*} \cdot \frac{\partial \Psi}{\partial q}\left(q_{*}, \dot{q}_{*}\right)\right)(t),
$$

with the inequality constraint

$$
\Phi\left(q_{*}, \dot{q}_{*}\right)(t)=\sigma_{*}(t)^{2} \geq 0
$$

the transversality condition

$$
\left(\frac{\partial \mathcal{L}}{\partial \dot{q}}\left(q_{*}, \dot{q}_{*}\right)+r_{*} \cdot \frac{\partial \Psi}{\partial \dot{q}}\left(q_{*}, \dot{q}_{*}\right)\right)(T)=0,
$$

and the complementarity condition

$$
r_{*}(t) \cdot \sigma_{*}(t)=0 .
$$

Proof. Define the Lagrangian $\hat{\mathcal{L}}$, where

$$
\hat{\mathcal{L}}(q, \dot{q}, r, \sigma) \equiv \mathcal{L}(q, \dot{q})+r \cdot\left(\Psi(q, \dot{q})-\sigma^{2}\right) .
$$

The Euler-Lagrange equations due to $q$ give us

$$
\frac{d}{d t} \frac{\partial \hat{\mathcal{L}}}{\partial \dot{q}}\left(q_{*}, \dot{q}_{*}, r_{*}, \sigma_{*}\right)=\frac{\partial \hat{\mathcal{L}}}{\partial q_{*}}\left(q_{*}, \dot{q}_{*}, r_{*}, \sigma_{*}\right)
$$

This simplifies to (4).

The Euler-Lagrange equations due to $r$ give us

$$
\frac{\partial \hat{\mathcal{L}}}{\partial r}\left(q_{*}, \dot{q}_{*}, r_{*}, \sigma_{*}\right)=0 \Longleftrightarrow \Psi\left(q_{*}, \dot{q}_{*}\right)=\sigma_{*}^{2} .
$$

Finally, the Euler-Lagrange equations due to $\sigma$ give us

$$
\frac{\partial \hat{\mathcal{L}}}{\partial \sigma}\left(q_{*}, \dot{q}_{*}, r_{*}, \sigma_{*}\right)=0 \Longleftrightarrow r_{*} \cdot \sigma_{*}=0,
$$

and we are done.

\subsection{Isoperimetric Constraints and Duality}

Finally, we model the optimization of a Lagrangian subjected to isoperimetric constraints.

Theorem 4 (Isoperimetric Constraints). If $\Theta: \mathbb{R}^{2} \rightarrow \mathbb{R}$ is a differentiable mapping, then

$$
\underset{\substack{q: q(t)=x \\ \int_{0}^{T} \Theta(q, \dot{q})(t) d t=0}}{\operatorname{opt}}\left[\int_{0}^{T} \mathcal{L}(q, \dot{q})(t) d t\right]=\underset{\substack{q: q(t)=x \\ b: b(0)=b(T)=0,}}{\operatorname{opt}}\left[\int_{0}^{T} \mathcal{L}(q, \dot{q})(t)+a(t) \cdot(\dot{b}(t)-\Theta(q, \dot{q})(t)) d t\right] .
$$

This optimization problem simplifies to

$$
\begin{aligned}
& \underset{q: q(t)=x}{\operatorname{opt}} \quad\left[\int_{0}^{T} \mathcal{L}(q, \dot{q})(t) d t\right]=\underset{q: q(t)=x}{\operatorname{opt}}\left[\int_{0}^{T}(\mathcal{L}-a \cdot \Theta)(q, \dot{q})(t) d t\right] . \\
& \int_{0}^{T} \Theta(q, \dot{q})(t) d t=0
\end{aligned}
$$


Moreover, the optimal path $q_{*}:[0, T] \rightarrow \mathbb{R}$ satisfies the Euler-Lagrange equations

$$
\frac{d}{d t}\left(\frac{\partial \mathcal{L}}{\partial \dot{q}}-a \cdot \frac{\partial \Theta}{\partial \dot{q}}\right)\left(q_{*}, \dot{q}_{*}\right)(t)=\left(\frac{\partial \mathcal{L}}{\partial q}-a \cdot \frac{\partial \Theta}{\partial q}\right)\left(q_{*}, \dot{q}_{*}\right)(t),
$$

with the transversality condition of

$$
\left(\frac{\partial \mathcal{L}}{\partial \dot{q}}-a \cdot \frac{\partial \Theta}{\partial \dot{q}}\right)\left(q_{*}, \dot{q}_{*}\right)(T)=0
$$

and $a$ is a constant that satisfies the isoperimetric condition of

$$
\int_{0}^{T} \Theta\left(q_{*}, \dot{q}_{*}\right)(t) d t=0 .
$$

Proof. Define the Lagrangian $\hat{\mathcal{L}}$, where

$$
\hat{\mathcal{L}}(a, \dot{b}, q, \dot{q}) \equiv \mathcal{L}(q, \dot{q})+a \cdot(\dot{b}-\Theta(q, \dot{q})),
$$

where we add the boundary conditions of $b(0)=b(T)=0$.

The Euler-Lagrange equations due to $q$ give us

$$
\frac{d}{d t} \frac{\partial \hat{\mathcal{L}}}{\partial \dot{q}}\left(a_{*}, \dot{b}_{*}, q_{*}, \dot{q}_{*}\right)=\frac{\partial \hat{\mathcal{L}}}{\partial q_{*}}\left(a_{*}, \dot{b}_{*}, q_{*}, \dot{q}_{*}\right) .
$$

This simplifies to (5). The transversality condition of (6) is similarly derived.

The Euler-Lagrange equations due to $a$ give us

$$
\frac{\partial \hat{\mathcal{L}}}{\partial a}\left(a_{*}, \dot{b}_{*}, q_{*}, \dot{q}_{*}\right)=0 \Longleftrightarrow \dot{b}_{*}=\Theta\left(q_{*}, \dot{q}_{*}\right)
$$

Integrating the latter equation over the interval $(0, T]$, combined with the conditions of $b(0)=b(T)=0$, gives us $(7)$.

Finally, the Euler-Lagrange equations due to $b$ give us

$$
\frac{d}{d t} \frac{\partial \hat{\mathcal{L}}}{\partial \dot{b}}\left(a_{*}, \dot{b}_{*}, q_{*}, \dot{q}_{*}\right)=0 \Longleftrightarrow \dot{a}_{*}=0 .
$$

This makes $a_{*}$ a constant over time, and we are done.

Problem 2 (Maximizing Area Constrained by Perimeter). As illustrated in Figure 3 , find the maximal area $|\Omega|$, for a simply connected region $\Omega$ in $\mathbb{R}^{2}$, bounded by a fixed perimeter $|\partial \Omega|$ of the boundary $\partial \Omega$.

Solution. Let $(x, y):(0, T] \rightarrow \partial \Omega$ be a one-to-one differentiable mapping and $(x, y)$ : $[0, T] \rightarrow \partial \Omega$ a differentiable mapping. Moreover, we assume that $x(0)=x(T), x^{\prime}(0+)=$ $x^{\prime}(T-)$, and $y$ has the same properties.

We can compute the perimeter of $\partial \Omega$ by using this parameterization of the boundary for $\Omega$ and get

$$
|\partial \Omega|=\int_{0}^{T} \sqrt{\dot{x}(t)^{2}+\dot{y}(t)^{2}} d t .
$$

We can also use the parameterization of the boundary curve $\partial \Omega$ to express the area of $\Omega$ as a line intergral. This yields

$$
|\Omega|=\frac{1}{2} \int_{0}^{T}(x(t) \dot{y}(t)-y(t) \dot{x}(t)) d t,
$$


FiguRE 3. Finding the maximum area given a fixed perimeter for the boundary.

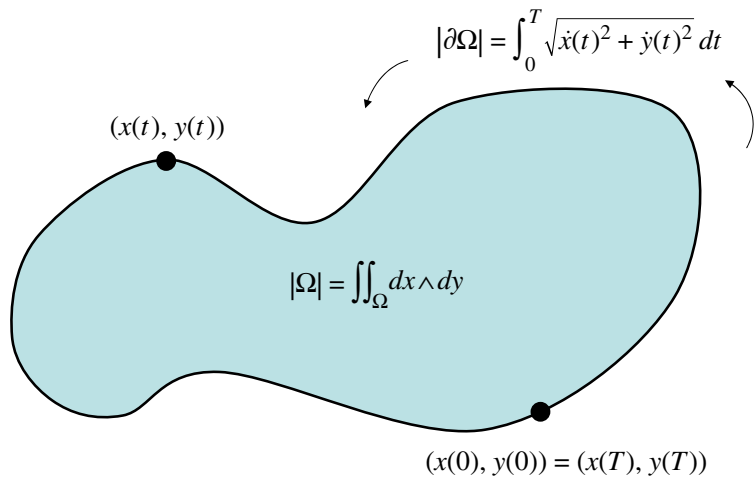

since by Green's theorem we have

$$
|\Omega|=\iint_{\Omega} d x \wedge d y=\frac{1}{2} \int_{\partial \Omega} x d y-y d x
$$

Now we have an optimization problem with an isoperimetric constraint. Our Lagrangian is

$$
\mathcal{L}(a, \dot{b}, x, \dot{x}, y, \dot{y})=\frac{1}{2}(x \dot{y}-y \dot{x})+a \cdot\left(\dot{b}-\sqrt{\dot{x}^{2}+\dot{y}^{2}}\right) .
$$

The Euler-Lagrange equations for $x$ are

$$
\frac{d}{d t} \frac{\partial \mathcal{L}}{\partial \dot{x}}=\frac{\partial \mathcal{L}}{\partial x} \Longleftrightarrow \frac{d}{d t}\left(-\frac{1}{2} y(t)-\frac{a \cdot \dot{x}(t)}{\sqrt{\dot{x}(t)^{2}+\dot{y}(t)^{2}}}\right)=\frac{1}{2} \dot{y}(t) .
$$

Similarly for $y$ we have

$$
\frac{d}{d t} \frac{\partial \mathcal{L}}{\partial \dot{y}}=\frac{\partial \mathcal{L}}{\partial y} \Longleftrightarrow \frac{d}{d t}\left(\frac{1}{2} x(t)-\frac{a \cdot \dot{y}(t)}{\sqrt{\dot{x}(t)^{2}+\dot{y}(t)^{2}}}\right)=-\frac{1}{2} \dot{x}(t) .
$$

For the state variable $b$, we have

$$
\frac{d}{d t} \frac{\partial \mathcal{L}}{\partial \dot{b}}=\frac{\partial \mathcal{L}}{\partial b}=0 \Longleftrightarrow \dot{a}(t)=0
$$

for all $0 \leq t \leq T$. This means that $a$ is a constant. Finally, for its dual variable $b$ we have

$$
0=\frac{d}{d t} \frac{\partial \mathcal{L}}{\partial \dot{a}}=\frac{\partial \mathcal{L}}{\partial a} \Longleftrightarrow \dot{b}(t)=\sqrt{\dot{x}(t)^{2}+\dot{y}(t)^{2}} .
$$

Coupled with the initial and terminal conditions of $b(0)=0$ and $b(T)=|\partial \Omega|$, we then have (8).

Simplifying (9) and (10) yields

$$
\frac{d}{d t}\left(-y(t)-\frac{a \cdot \dot{x}(t)}{\sqrt{\dot{x}(t)^{2}+\dot{y}(t)^{2}}}\right)=0 \quad \text { and } \quad \frac{d}{d t}\left(x(t)-\frac{a \cdot \dot{y}(t)}{\sqrt{\dot{x}(t)^{2}+\dot{y}(t)^{2}}}\right)=0 .
$$

This means that there exists two constants $\xi$ and $\eta$ such that

$$
y(t)-\eta=\frac{a \cdot \dot{x}(t)}{\sqrt{\dot{x}(t)^{2}+\dot{y}(t)^{2}}} \quad \text { and } \quad x(t)-\xi=\frac{a \cdot \dot{y}(t)}{\sqrt{\dot{x}(t)^{2}+\dot{y}(t)^{2}}} .
$$

From this it follows that $x(t)$ and $y(t)$ solve the algebraic equation

$$
(x(t)-\xi)^{2}+(y(t)-\eta)^{2}=a^{2} .
$$

This means that $\Omega$ is a circle of radius $a$. The last step is to select $a$ such that $|\partial \Omega|=2 \pi a$. 
We can apply these same arguments to solve a dual problem.

Problem 3 (Minimizing Perimeter Constrained by Area). Find the minimal perimeter $|\partial \Omega|$, for the boundary $\partial \Omega$ of a simply connected region $\Omega$ in $\mathbb{R}^{2}$, given a fixed area $|\Omega|$.

Suppose that we start with a given area value. Solving Problem 3 yields the minimal perimeter for this area. Conversely, if we assume this minimizing perimeter value, then the maximal area from the solution to Problem 2 is precisely the originally given area value. This is the continuous state version of the duality principle for linear programming. Here, the concepts of area and perimeter implicitly give us quantities that are always positive.

Below, we give a more generalized notion of this duality.

Theorem 5. Suppose that we have Lagrangians $\mathcal{L}$ and $\mathcal{M}$ such that:

(1) For all constants $x$, there exists an optimal path $\left\{q_{*}^{x}(t) \mid t \geq 0\right\}$ such that

$$
f(x) \equiv \int_{0}^{T}(\mathcal{L}-x \cdot \mathcal{M})\left(q_{*}^{x}, \dot{q}_{*}^{x}\right)(t) d t=\max _{q} \int_{0}^{T}(\mathcal{L}-x \cdot \mathcal{M})(q, \dot{q})(t) d t .
$$

(2) The function $f$ is convex with $f(0)>0, f^{\prime}(0)<0$, and

$$
\min _{x>0} f(x)=0 .
$$

It follows that

$$
\max _{q: \int_{0}^{T} \mathcal{M}(q, \dot{q})(t) d t=0} \int_{0}^{T} \mathcal{L}(q, \dot{q})(t) d t=\min _{q: \int_{0}^{T} \mathcal{L}(q, \dot{q})(t) d t=0} \int_{0}^{T} \mathcal{M}(q, \dot{q})(t) d t=0
$$

and

$$
\min _{x>0}\left(\max _{q} \int_{0}^{T}(\mathcal{L}-x \cdot \mathcal{M})(q, \dot{q})(t) d t\right)=\max _{x>0}\left(\min _{q} \int_{0}^{T}(\mathcal{M}-x \cdot \mathcal{L})(q, \dot{q})(t) d t\right)=0 .
$$

Proof. Given the sensitivity results, we have

$$
f^{\prime}(x)=-\int_{0}^{T} \mathcal{M}\left(q_{*}^{x}, \dot{q}_{*}^{x}\right)(t) d t=0 .
$$

The conditions of $f(0)>0$ and $f^{\prime}(0)<0$ translate into

$$
\int_{0}^{T} \mathcal{L}\left(q_{*}^{0}, \dot{q}_{*}^{0}\right)(t) d t=\max _{q} \int_{0}^{T} \mathcal{L}(q, \dot{q})(t) d t>0
$$

and

$$
\int_{0}^{T} \mathcal{M}\left(q_{*}^{0}, \dot{q}_{*}^{0}\right)(t) d t>0 .
$$

Starting from zero, $f$ is a decreasing function as long as $f^{\prime}(x)>0$. Since there exists an $x_{*}>0$ with $f\left(x_{*}\right)=0$, we then have $f^{\prime}\left(x_{*}\right)=0$ and so

$$
\max _{q: \int_{0}^{T} \mathcal{M}(q, \dot{q})(t) d t=0} \int_{0}^{T} \mathcal{L}(q, \dot{q})(t) d t=\min _{x>0} f(x)=0 .
$$

Now $\left\{q_{*}^{x}(t) \mid t \geq 0\right\}$ solves the Euler-Lagrange equations for $\mathcal{L}-x \cdot \mathcal{M}$. Since

$$
\mathcal{M}-\frac{1}{x_{*}} \cdot \mathcal{L}=-\frac{1}{x} \cdot(\mathcal{L}-x \cdot \mathcal{M}),
$$

it follows that $\left\{q_{*}^{x}(t) \mid t \geq 0\right\}$ also solves the Euler-Lagrange equations for $\mathcal{M}-1 / x_{*} \cdot \mathcal{L}$. 
Now we define the function $g(x)$, where

$$
g(x) \equiv \min _{q} \int_{0}^{T}(\mathcal{L}-x \cdot \mathcal{M})(q, \dot{q})(t) d t .
$$

Based on our assumptions, there exists an $x_{*}$ such that $f\left(x_{*}\right)=f^{\prime}\left(x_{*}\right)=0$. For all positive $x$, we then have

$$
g(x)=-x \cdot f\left(\frac{1}{x}\right)
$$

Through various operations such as differentiating and multiplying by $x$, we obtain

$$
g^{\prime}(x)=-f\left(\frac{1}{x}\right)-\frac{1}{x} \cdot f\left(\frac{1}{x}\right) \quad \text { and } \quad g^{\prime \prime}(x)=-f^{\prime \prime}\left(\frac{1}{x}\right) .
$$

It now follows that $g$ is a negative, concave function with $g\left(1 / x_{*}\right)=g^{\prime}\left(1 / x_{*}\right)=0$; hence $g$ attains its maximum at $1 / x_{*}$ and

$$
g\left(1 / x_{*}\right)=\max _{x>0} g(x)=0 .
$$

\subsection{Conserved Quantities}

We conclude this section by showing how to construct quantities that are conserved along the optimal Lagrangian path. The following result is adapted from Sato and Ramachandran [44].

Theorem 6. Let $\theta$ parameterize a differentiable transformation from $(q(t), t)$ to $\left(q_{\theta}\left(t_{\theta}\right), t_{\theta}\right)$, where

$$
q_{\theta}\left(t_{\theta}\right)=\mathfrak{q}_{\theta}(q(t), \dot{q}(t), t) \quad \text { and } \quad t_{\theta}=\mathfrak{t}_{\theta}(q(t), \dot{q}(t), t),
$$

with $q(t)=q_{0}\left(t_{0}\right)$ and $t=t_{0}$. If we have

$$
\frac{d}{d t} \mathcal{G}(q(t), t)=\left.\frac{d}{d \theta}\left(\mathcal{L}\left(q_{\theta}\left(t_{\theta}\right), \dot{q}_{\theta}\left(t_{\theta}\right), t_{\theta}\right) \cdot \frac{d}{d t} t_{\theta}\right)\right|_{\theta=0},
$$

then the deterministic process

$$
\mathcal{G}(q)-\frac{\partial \mathcal{L}}{\partial \dot{q}}(q, \dot{q}) \cdot \hat{\mathfrak{q}}(q, \dot{q})-\left(\mathcal{L}(q, \dot{q})-\frac{\partial \mathcal{L}}{\partial \dot{q}}(q, \dot{q}) \cdot \dot{q}\right) \cdot \hat{\mathfrak{t}}(q, \dot{q})
$$

is a conserved quantity along the optimal path, where

$$
\left.\hat{\mathfrak{q}}(q, \dot{q}) \equiv \frac{d}{d \theta} \mathfrak{q}_{\theta}(q, \dot{q})\right|_{\theta=0} \quad \text { and }\left.\quad \hat{\mathfrak{t}}(q, \dot{q}) \equiv \frac{d}{d \theta} \mathfrak{t}_{\theta}(q, \dot{q})\right|_{\theta=0} .
$$

Proof. Observe that

$$
\dot{q}_{\theta}\left(t_{\theta}\right)=\frac{d}{d t} \mathfrak{q}_{\theta}(q(t), \dot{q}(t), t) / \frac{d}{d t} \mathfrak{t}_{\theta}(q(t), \dot{q}(t), t)=\dot{\mathfrak{q}}_{\theta}(q(t), \dot{q}(t), t) / \dot{\mathfrak{t}}_{\theta}(q(t), \dot{q}(t), t) .
$$

This means that we can rewrite (11) as

$$
\dot{\mathcal{G}}(q)=\left.\frac{d}{d \theta} \mathcal{L}\left(\mathfrak{q}_{\theta}(q, \dot{q}), \dot{\mathfrak{q}}_{\theta}(q, \dot{q}) / \dot{\mathfrak{t}}_{\theta}(q, \dot{q}), \mathfrak{t}_{\theta}(q, \dot{q})\right) \cdot \dot{\mathfrak{t}}_{\theta}(q, \dot{q})\right|_{\theta=0}
$$

Along the optimal path, the Euler-Lagrange equations hold, so we have

$$
\frac{d}{d t} \frac{\partial \mathcal{L}}{\partial \dot{q}}\left(q_{*}(t), \dot{q}_{*}(t), t\right)=\frac{\partial \mathcal{L}}{\partial q}\left(q_{*}(t), \dot{q}_{*}(t), t\right) .
$$


The rest follows from the computation below:

$$
\begin{aligned}
\frac{d}{d \theta}( & \left.\mathcal{L}\left(\mathfrak{q}_{\theta}\left(q_{*}, \dot{q}_{*}\right), \dot{\mathfrak{q}}_{\theta}\left(q_{*}, \dot{q}_{*}\right) / \dot{\mathfrak{t}}_{\theta}\left(q_{*}, \dot{q}_{*}\right), \mathfrak{t}_{\theta}\left(q_{*}, \dot{q}_{*}\right)\right) \cdot \dot{\mathfrak{t}}_{\theta}\left(q_{*}, \dot{q}_{*}\right)\right)\left.\right|_{\theta=0} \\
= & \frac{\partial \mathcal{L}}{\partial q}\left(q_{*}, \dot{q}_{*}\right) \cdot \hat{\mathfrak{q}}\left(q_{*}, \dot{q}_{*}\right)+\frac{\partial \mathcal{L}}{\partial \dot{q}}\left(q_{*}, \dot{q}_{*}\right) \cdot\left(\dot{\hat{\mathfrak{q}}}\left(q_{*}, \dot{q}_{*}\right)-\dot{q}_{*} \cdot \dot{\hat{\mathfrak{t}}}\left(q_{*}, \dot{q}_{*}\right)\right) \\
& +\frac{\partial \mathcal{L}}{\partial t}\left(q_{*}, \dot{q}_{*}\right) \cdot \hat{\mathfrak{t}}\left(q_{*}, \dot{q}_{*}\right)+\mathcal{L}\left(q_{*}, \dot{q}_{*}\right) \cdot \dot{\hat{\mathfrak{t}}}\left(q_{*}, \dot{q}_{*}\right) \\
= & \frac{d}{d t}\left(\frac{\partial \mathcal{L}}{\partial \dot{q}}\left(q_{*}, \dot{q}_{*}\right)\right) \cdot \hat{\mathfrak{q}}\left(q_{*}, \dot{q}_{*}\right)+\frac{\partial \mathcal{L}}{\partial \dot{q}}\left(q_{*}, \dot{q}_{*}\right) \cdot \dot{\hat{\mathfrak{q}}}\left(q_{*}, \dot{q}_{*}\right)-\frac{\partial \mathcal{L}}{\partial \dot{q}}\left(q_{*}, \dot{q}_{*}\right) \cdot \dot{q}_{*} \cdot \dot{\hat{\mathfrak{t}}}\left(q_{*}, \dot{q}_{*}\right) \\
& +\frac{d}{d t}\left(\mathcal{L}\left(q_{*}, \dot{q}_{*}\right)-\frac{\partial \mathcal{L}}{\partial \dot{q}}\left(q_{*}, \dot{q}_{*}\right) \cdot \dot{q}_{*}\right) \cdot \hat{\mathfrak{t}}\left(q_{*}, \dot{q}_{*}\right)+\mathcal{L}\left(q_{*}, \dot{q}_{*}\right) \cdot \dot{\hat{\mathfrak{t}}}\left(q_{*}, \dot{q}_{*}\right) \\
& \frac{d}{d t}\left(\frac{\partial \mathcal{L}}{\partial \dot{q}}\left(q_{*}, \dot{q}_{*}\right)\right) \cdot \hat{\mathfrak{q}}\left(q_{*}, \dot{q}_{*}\right)+\frac{\partial \mathcal{L}}{\partial \dot{q}}\left(q_{*}, \dot{q}_{*}\right) \cdot \dot{\hat{\mathfrak{q}}}\left(q_{*}, \dot{q}_{*}\right) \\
& +\frac{d}{d t}\left(\mathcal{L}\left(q_{*}, \dot{q}_{*}\right)-\frac{\partial \mathcal{L}}{\partial \dot{q}}\left(q_{*}, \dot{q}_{*}\right) \cdot \dot{q}_{*}\right) \cdot \hat{\mathfrak{t}}\left(q_{*}, \dot{q}_{*}\right)+\left(\mathcal{L}\left(q_{*}, \dot{q}_{*}\right)-\frac{\partial \mathcal{L}}{\partial \dot{q}}\left(q_{*}, \dot{q}_{*}\right) \cdot \dot{q}_{*}\right) \cdot \dot{\hat{\mathfrak{t}}}\left(q_{*}, \dot{q}_{*}\right) \\
= & \frac{d}{d t}\left(\frac{\partial \mathcal{L}}{\partial \dot{q}}\left(q_{*}, \dot{q}_{*}\right) \cdot \hat{\mathfrak{q}}\left(q_{*}, \dot{q}_{*}\right)+\left(\mathcal{L}\left(q_{*}, \dot{q}_{*}\right)-\frac{\partial \mathcal{L}}{\partial \dot{q}}\left(q_{*}, \dot{q}_{*}\right) \cdot \dot{q}_{*}\right) \cdot \hat{\mathfrak{t}}\left(q_{*}, \dot{q}_{*}\right)\right) \cdot \square
\end{aligned}
$$

\section{The Hamiltonian}

From the perspective of classical mechanics, the Lagrangian through the Euler-Lagrange equations successfully captured the laws of Newtonian physics. However, quantities like it and its time integral called "action" had no physical interpretation. They were merely mathematical formalisms that worked. One could attribute this to the fact that philosophically classical physics was not thought of in terms of quantities that are optimized. This is in contrast to the operations research and economic views of the Lagrangian. Here, we can interpret the Lagrangian as a "value rate" since operations researchers and economists naturally maximize quantities like "profit" or "revenue" and minimize quantities like "loss" or "cost."

We formally define the Hamiltonian in terms of the Lagrangian to be

$$
\mathcal{H}(p, q, t) \equiv \overline{v \in \mathbb{R}}(p \cdot v-\mathcal{L}(q, v, t))
$$

The optimization method that yields the Hamiltonian is called the Legendre transform. In the next section we study its special properties. Instead of using the optimal paths and velocities of $q_{*}$ and $\dot{q}_{*}$, we keep the former but replace the latter with a generalized momentum variable $p_{*}$. This allows us to replace the Euler-Lagrange equations for position and velocity with the equivalent set of Hamilton's equations for position and momentum. Unlike the Euler-Lagrange equations, Hamilton's equations are always dynamical systems. These are evolution equations that lend themselves to computationally simple numerical solutions. Finally, the Hamiltonian perspective helps us identify conserved quantities. In addition to terms like energy and momentum, there may be other conservation principles at work for a given Hamiltonian system.

\subsection{Legendre Transforms}

First, for some function $l: \mathbb{R} \rightarrow \mathbb{R}$, we define the Legendre transform (when it exists) to be the function $h: \mathbb{R} \rightarrow \mathbb{R}$, such that

$$
h(p) \equiv \underset{v \in \mathbb{R}}{\operatorname{opt}}(p \cdot v-l(v)) .
$$


Figure 4. The Legendre transform for the case of opt $=\max$.

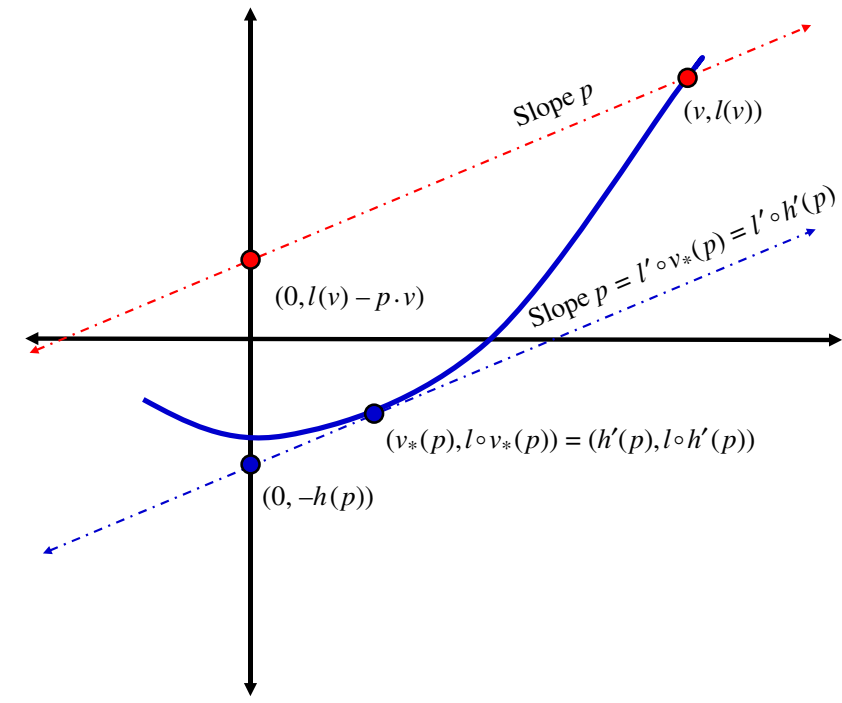

For the case of opt being max, an economic interpretation follows by defining $l(v)$ to be the cost of producing the volume (or total number of items sold) of $v$ items. Now let $p$ be the price of each item sold. The Legendre transform $h(p)$ is optimal profit that is obtained with the optimal volume of items produced, if we assume that every produced item is sold. Moreover, this optimal volume equals $h^{\prime}(p)$. This is the instantaneous rate of change in profit with respect to a unit change in the price.

In Figure 4, we can also give a geometric interpretation of the Legendre transformation when opt is max. For a given slope $p$, we want to find the "smallest" line of that slope to intersect with the graph of $l$. The negative of the $y$-intercept of that minimal line equals $h(p)$.

For the case of Newtonian mechanics as given in Example 1, the integral of the Lagrangian is something that is minimized. Thus, opt $=\min , \overline{\mathrm{opt}}=\max$, and the corresponding Hamiltonian is

$$
\begin{aligned}
\mathcal{H}(p, q)(t) & =\max _{v \in \mathbb{R}}(p \cdot v-\mathcal{L}(q, v, t)) \\
& =\max _{v \in \mathbb{R}}\left(p \cdot v-\frac{1}{2} m v^{2}+V(q)\right) \\
& =\max _{v \in \mathbb{R}}\left(p \cdot v-\frac{1}{2} m v^{2}\right)+V(q) \\
& =\max _{v \in \mathbb{R}}\left(\frac{p^{2}}{2 m}-\frac{m}{2}\left(v-\frac{p}{m}\right)^{2}\right)+V(q) \\
& =\frac{p^{2}}{2 m}+V(q) .
\end{aligned}
$$

Moreover, the maximum value for the Hamiltonian is achieved when $p=m v$, since $m>0$.

\subsection{Hamilton's Equations}

We begin this section by continuing our discussion of Example 1. If we have $\mathcal{L}\left(q_{*}, \dot{q}_{*}\right)(t)=$ $p_{*} \cdot \dot{q}_{*}-\mathcal{H}\left(p_{*}, q_{*}\right)(t)$, then

$$
p_{*}=m \cdot \dot{q}_{*}=\frac{\partial \mathcal{L}}{\partial \dot{q}_{*}}\left(q_{*}, \dot{q}_{*}\right)(t) ;
$$


hence

$$
\frac{\partial \mathcal{H}}{\partial p}\left(p_{*}, q_{*}\right)(t)=\frac{p_{*}}{m}=\dot{q}_{*} \quad \text { and } \quad \frac{\partial \mathcal{H}}{\partial q}\left(p_{*}, q_{*}\right)(t)=V^{\prime}\left(q_{*}\right)=-\dot{p}_{*} .
$$

Finally, observe that if the mass $m$ is constant, then

$$
\frac{d}{d t} \mathcal{H}\left(p_{*}, q_{*}\right)(t)=\frac{p_{*}}{m} \cdot \dot{p}_{*}+V^{\prime}\left(q_{*}\right) \cdot \dot{q}_{*}=\dot{q}_{*} \cdot \dot{p}_{*}-\dot{p}_{*} \cdot \dot{q}_{*}=0 .
$$

Thus along the optimum path, $\mathcal{H}\left(p_{*}, q_{*}\right)(t)$ is a constant over time.

We now summarize these results in a larger framework.

Theorem 7 (Hamilton's Equations). If we have

$$
\mathcal{H}\left(p_{*}, q_{*}\right)(t)=p_{*}(t) \cdot \dot{q}_{*}(t)-\mathcal{L}\left(q_{*}, \dot{q}_{*}\right)(t),
$$

then

$$
p_{*}(t)=\frac{\partial \mathcal{L}}{\partial \dot{q}}\left(q_{*}, \dot{q}_{*}\right)(t) .
$$

Moreover, we have

$$
\dot{p}_{*}(t)=-\frac{\partial \mathcal{H}}{\partial q}\left(p_{*}, q_{*}\right)(t) \quad \text { and } \quad \dot{q}_{*}(t)=\frac{\partial \mathcal{H}}{\partial p}\left(p_{*}, q_{*}\right)(t) .
$$

Proof. Our definition of the Hamiltonian means that we have some $v_{*}(p, q, t)$ such that

$$
\mathcal{H}(p, q, t)=p \cdot v_{*}(p, q, t)-\mathcal{L}\left(q, v_{*}(p, q, t), t\right) .
$$

Now we make the substitutions

$$
p=p_{*}(t), \quad q=q_{*}(t), \quad \text { and } \quad v_{*}\left(p_{*}, q_{*}\right)(t)=\dot{q}_{*}(t) .
$$

The Equation (14) follows from (13), since

$$
0=\left.\frac{\partial}{\partial v}\right|_{v_{*}(p, q, t)}(p \cdot v-\mathcal{L}(q, v, t)) \quad \text { implies that } \quad p=\frac{\partial \mathcal{L}}{\partial \dot{q}}\left(q, v_{*}(p, q, t), t\right) .
$$

The computation of the partial derivatives of the Hamiltonian with respect to $p$ and $q$ in (16) simplifies considerably due to the envelope lemma (see Lemma 1). This result allows us to ignore the dependence of $v(p, q)$ on $p$ and $q$ and state that

$$
\frac{\partial \mathcal{H}}{\partial p}(p, q, t)=v_{*}(p, q, t) \quad \text { and } \quad \frac{\partial \mathcal{H}}{\partial q}(p, q, t)=-\frac{\partial \mathcal{L}}{\partial q}\left(q, v_{*}(p, q, t), t\right) .
$$

Now Hamilton's equations (15) follow from applying the substitutions of (17), (14), and the Euler-Lagrange equations (1).

\subsection{Computing Hamiltonian Flows}

Given a Lagrangian $\mathcal{L}$ and an optimal position coordinate $q_{*}$ yields a Hamiltonian $\mathcal{H}$ and what we define to be an optimal momentum coordinate $p_{*}$ given by (14). The coordinate pair of $p_{*}$ and $q_{*}$ describes a point in phase space. The evolution of these coordinates over time $\left\{\left(p_{*}(t), q_{*}(t)\right) \mid 0 \leq t \leq T\right\}$ is called a Hamiltonian flow. We now state an algorithm for computing this flow (see Figure 5). Initially, we assume that

$$
q_{0}(t)=q(0)
$$

for all $0 \leq t \leq T$. Now we iterate through time loops that start at time $T$, go back to the past of time 0 , and then go back to the future of time $T$. Recursively, the $n$th time loop, 
FiguRE 5. A flow chart diagram for computing Hamilton's equations.

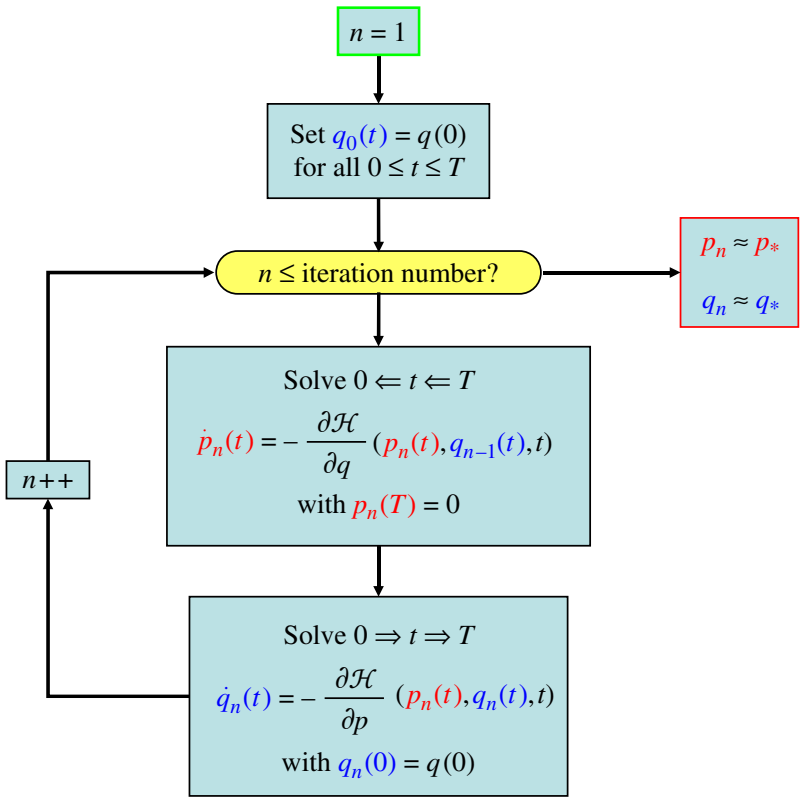

where $n \geq 1$, is

(1) Given $\left\{q_{n-1}(t) \mid 0 \leq t \leq T\right\}$, solve for the dynamical system

$$
\dot{p}_{n}(t)=-\frac{\partial \mathcal{H}}{\partial q}\left(p_{n}, q_{n-1}\right)(t)
$$

backward in time for $0 \leq t \leq T$, starting with the terminal condition $p_{n}(T)=0$.

(2) Given $\left\{p_{n}(t) \mid 0 \leq t \leq T\right\}$, solve for the dynamical system

$$
\dot{q}_{n}(t)=\frac{\partial \mathcal{H}}{\partial p}\left(p_{n}, q_{n}\right)(t)
$$

forward in time for $0 \leq t \leq T$, starting with the initial condition $q_{n}(0)=q(0)$.

\subsection{Poisson Brackets and Conservation Principles}

Corollary 3 (Conservation of Momentum). Along the Hamiltonian flow, we have

$$
\frac{\partial \mathcal{L}}{\partial q}\left(p_{*}, q_{*}\right)(t)=0 \quad \text { if and only if } \frac{\partial \mathcal{H}}{\partial q}\left(p_{*}, q_{*}\right)(t)=0 .
$$

Moreover, both statements are equivalent to $\dot{p}_{*}(t)=0$.

Proof. Using the Euler-Lagrange equations (1) and Hamilton's equations (15) yields

$$
\dot{p}_{*}(t)=\frac{\partial \mathcal{L}}{\partial q}\left(p_{*}, q_{*}\right)(t)=-\frac{\partial \mathcal{H}}{\partial q}\left(p_{*}, q_{*}\right)(t) .
$$

Corollary 4 (Conservation of Energy). Along the Hamiltonian flow, whenever we have

$$
\frac{\partial \mathcal{H}}{\partial t}\left(p_{*}, q_{*}\right)(t)=0
$$

it follows that

$$
\frac{d}{d t} \mathcal{H}\left(p_{*}, q_{*}\right)(t)=0
$$


Proof. Using Theorem 7 yields

$$
\begin{aligned}
\frac{d}{d t} \mathcal{H}\left(p_{*}, q_{*}\right)(t) & =\frac{\partial \mathcal{H}}{\partial p}\left(p_{*}, q_{*}\right)(t) \cdot \dot{p}_{*}(t)+\frac{\partial \mathcal{H}}{\partial q}\left(p_{*}, q_{*}\right)(t) \cdot \dot{q}_{*}(t)+\frac{\partial \mathcal{H}}{\partial t}\left(p_{*}, q_{*}\right)(t) \\
& =\dot{q}_{*}(t) \cdot \dot{p}_{*}(t)-\dot{p}_{*}(t) \cdot \dot{q}_{*}(t) \\
& =0 . \quad \square
\end{aligned}
$$

These two results are a part of a larger conservation principle. We define an observable for the Hamiltonian flow to be any differentiable mapping $\Phi: \mathbb{R}^{2} \times[0, T] \rightarrow \mathbb{R}$. Now we describe the dynamics of $\Phi\left(p_{*}, q_{*}\right)$.

Corollary 5 (Poisson Brackets). Given a Hamiltonian $\mathcal{H}$, let $p_{*}$ and $q_{*}$ be optimal paths that solve Hamilton's equations (15). If $\Phi$ is an observable, then

$$
\frac{d}{d t} \Phi\left(p_{*}, q_{*}\right)(t)=\left(\{\Phi, \mathcal{H}\}+\frac{\partial \Phi}{\partial t}\right)\left(p_{*}, q_{*}\right)(t),
$$

where $\{\cdot, \cdot\}$ is the Poisson bracket that is defined by the formula

$$
\{\Phi, \Psi\}(p, q, t) \equiv\left(\frac{\partial \Phi}{\partial q} \cdot \frac{\partial \Psi}{\partial p}-\frac{\partial \Psi}{\partial q} \cdot \frac{\partial \Phi}{\partial p}\right)(p, q, t),
$$

where $\Psi$ is another observable.

Proof. Applying the chain rule for the total differential with respect to $t$ yields

$$
\begin{aligned}
\frac{d}{d t} \Phi\left(p_{*}, q_{*}\right)(t) & =\frac{\partial \Phi}{\partial p}\left(p_{*}, q_{*}\right)(t) \cdot \dot{p}_{*}(t)+\frac{\partial \Phi}{\partial q}\left(p_{*}, q_{*}\right)(t) \cdot \dot{q}_{*}(t)+\frac{\partial \Phi}{\partial t}\left(p_{*}, q_{*}\right)(t) \\
& =\left(-\frac{\partial \Phi}{\partial p} \cdot \frac{\partial \mathcal{H}}{\partial q}+\frac{\partial \Phi}{\partial q} \cdot \frac{\partial \mathcal{H}}{\partial p}+\frac{\partial \Phi}{\partial t}\right)\left(p_{*}, q_{*}\right)(t)
\end{aligned}
$$

Now we apply (18) to (19), and we are done.

The Poisson bracket also has various algebraic properties. First, it is antisymmetric; i.e., for all observables $\Phi$ and $\Psi$, we have

$$
\{\Phi, \Psi\}=-\{\Psi, \Phi\}
$$

Moreover, it is also a bilinear operation, with

$$
\{\Phi+\Psi, \mathcal{H}\}=\{\Phi, \mathcal{H}\}+\{\Psi, \mathcal{H}\}
$$

and

$$
\{a \cdot \Phi, \mathcal{H}\}=a \cdot\{\Phi, \mathcal{H}\} .
$$

Finally, the Poisson bracket satisfies the Jacobi identity or

$$
\{\{\Phi, \Psi\}, \mathcal{H}\}=\{\Phi,\{\Psi, \mathcal{H}\}\}-\{\Psi,\{\Phi, \mathcal{H}\}\} .
$$

The Poisson bracket has the following geometric interpretation for two observables that are not time dependent or $\Phi$ and $\Psi$ where

$$
\frac{\partial \Phi}{\partial t}=\frac{\partial \Psi}{\partial t}=0
$$

In Figure 6, we fix $t$ and view $\Phi$ and $\Psi$ as scalar fields over the "phase space" given by the momentum and position coordinates of $p$ and $q$, respectively. The dotted curve represents a contour for $\Phi$ or the set of phase-space coordinates where it equals some constant $a$. The solid curve plays a similar role as the contour for $\Psi$ equalling a constant $b$. The gradient of $\Phi$ is 
FiguRE 6. A geometric interpretation of the Poisson bracket.

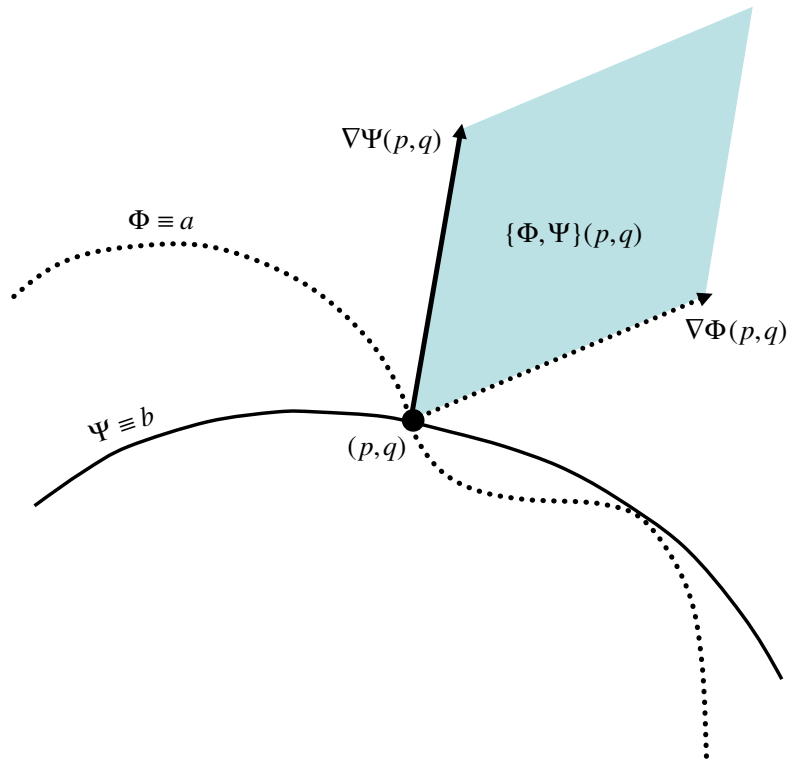

the direction of greatest change for its scalar field. It is always orthogonal to the tangent line for the contour curve at that point $(p, q)$. Locally, this means that the gradient is orthogonal to the direction that "conserves $\Phi$." The gradient for $\Psi$ has a similar interpretation. The Poisson bracket is then the area (up to a sign for orientation) of the parallelogram formed by the two gradients at a given point $(p, q)$ in phase space. This area is zero if and only if the two gradients are colinear or they "point in the same direction" (up to a sign). When the Poisson bracket is zero along the contour of one of them, then the same curve is a contour for the other and it is possible to move through phase space in a direction that conserves both observables $\Phi$ and $\Psi$. For more on the algebraic structure of Hamiltonian dynamics, see Marsden and Ratiu [34].

We define an observable $\Phi$ to be a conserved quantity over the interval $[0, T]$ if

$$
\frac{d}{d t} \Phi\left(p_{*}, q_{*}\right)(t)=0
$$

Now we can characterize the underlying structure of conserved quantities.

Corollary 6. An observable $\Phi$ is a conserved quantity over the interval $[0, T]$ whenever

$$
\left(\frac{\partial \Phi}{\partial t}+\{\Phi, \mathcal{H}\}\right)\left(p_{*}, q_{*}\right)(t)=0
$$

for all $0 \leq t \leq T$. Moreover, the set of conserved quantities form a vector space that is also closed with respect to the Poisson bracket operation.

Proof. Given two conserved quantities $\Phi$ and $\Psi$, we have

$$
\begin{aligned}
\frac{\partial}{\partial t}\{\Phi, \Psi\} & =\left\{\frac{\partial \Phi}{\partial t}, \Psi\right\}+\left\{\Phi, \frac{\partial \Psi}{\partial t}\right\} \\
& =-\{\{\Phi, \mathcal{H}\}, \Psi\}-\{\Phi,\{\Psi, \mathcal{H}\}\} \\
& =\{\Psi,\{\Phi, \mathcal{H}\}\}-\{\Phi,\{\Psi, \mathcal{H}\}\} \\
& =\{\{\Psi, \Phi\}, \mathcal{H}\} \\
& =-\{\{\Phi, \Psi\}, \mathcal{H}\} .
\end{aligned}
$$

The third step follows from the Jacobi identity. 
We have now shown that the family of conserved quantities form a Lie algebra with respect to addition and the Poisson bracket operation as our anticommutative, nonassociative multiplication. If we are given two or more conserved quantities, we can then use linear combinations of them and the Poisson bracket to generate the associated Lie algebra of additional conserved quantities.

For operations research problems, the Hamiltonian formulation is useful from a computational perspective. It is easier to numerically solve differential equations that are dynamical systems than some of the Euler-Lagrange equations derived from Lagrangian mechanics. This simplicity is somewhat compromised by the mixed boundary values of an initial value for $q$ paired with a terminal value of $p(T)=0$. However, the dynamical system structure suggests an iterative method for the solution. The nature of the method suggests evolving forwards in time with $q$ but backward in time with $p$. The Hamiltonian formulation also provides us with a Lie algebra calculus to derive conserved quantities.

\subsection{Envelope Lemma}

We conclude this section with a precise statement and proof of the envelope lemma.

Lemma 1 (Envelope Lemma). Let $\mathcal{O} \subseteq \mathbb{R}^{2}$ be open and $f: \mathcal{O} \rightarrow \mathbb{R}$ be differentiable. If for all $x$, there exists some $\phi(x)$ such that

$$
f(x, \phi(x))=\underset{-\infty<y<\infty}{\text { opt }} f(x, y),
$$

then

$$
\frac{d}{d x} f(x, \phi(x))=\frac{\partial f}{\partial x}(x, \phi(x)) .
$$

Proof. Since $f$ is differentiable, then by (19) we have

$$
\frac{\partial f}{\partial y}(x, \phi(x))=0
$$

The rest follows by taking the total derivative of $f(x, \phi(x))$ with respect to $x$ and using the chain rule.

\section{The Bellman Value Function}

The Bellman value function can be defined in terms of the Lagrangian as follows:

$$
\mathcal{V}(x, t) \equiv \operatorname{opt}_{q: q(t)=x}\left[\int_{t}^{T} \mathcal{L}(q, \dot{q})(s) d s\right] .
$$

We show in this section how the Bellman value function yields a unifying framework for interpreting Lagrangians and Hamiltonians from an operations research perspective.

\subsection{Bellman Principle of Optimality}

Rather than derive the Euler-Lagrange equations, we can find the optimal path by using the equivalent fundamental principle of optimality due to Bellman (see Pedregal [40]), where

$$
\mathcal{V}\left(q_{*}\right)(t)=\underset{q: q(t)=x}{\operatorname{opt}}\left[\int_{t}^{\tau} \mathcal{L}(q, \dot{q})(s) d s+\mathcal{V}(q)(\tau)\right],
$$

for all intermediate times $\tau$ where $t \leq \tau \leq T$. 
Figure 7. Bellman principle of optimality.

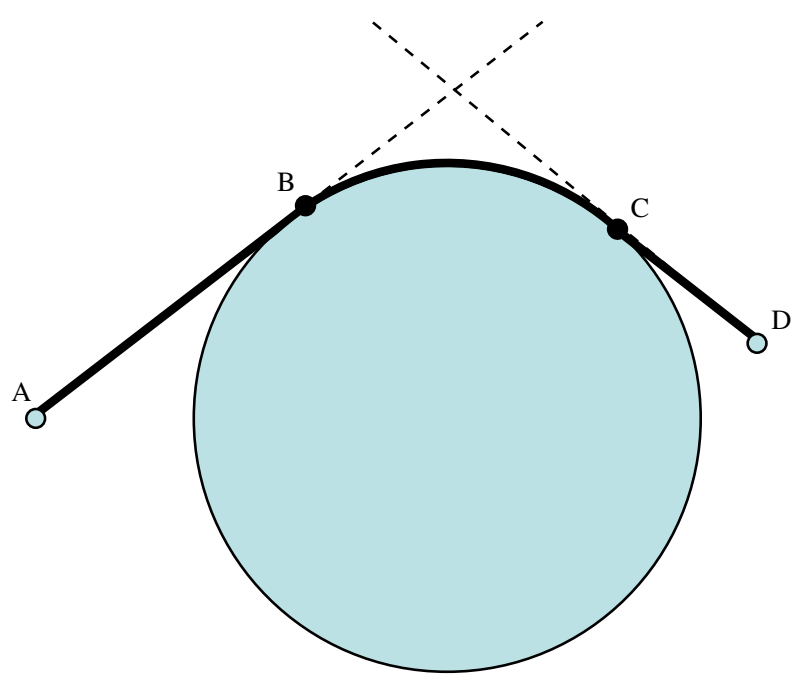

Problem 4 (Shortest Path Around an Obstacle). Consider two points in the Euclidean plane. They are denoted by A and D, as illustrated in Figure 7. The shortest path between them that is constrained to stay outside the disk is given by the unions of the lines $\mathrm{AB}$ and $\mathrm{CD}$ with is shortest of the circular arcs BC.

Solution. A simple way to show that this is the minimum path is by applying the Bellman principle. The subset of the path that connects $\mathrm{C}$ and $\mathrm{D}$ must be a straight line since that is the shortest distance in general and it is a line segment that is outside the disk. A similar argument can be made for why the subset of the path that connects $\mathrm{A}$ and $\mathrm{B}$ is also a straight line. Finally, we observe that a circle is defined to have the maximum area for a given perimeter. This means that the smaller of the two circular arcs connecting $\mathrm{B}$ and $\mathrm{C}$ is the minimum distance between the two points when the path is constrained to stay outside the disk.

\subsection{Hamilton-Jacobi-Bellman Equation}

The Bellman value function is defined in terms of the Lagrangian. In this section, we show how along the optimal path we can use the Bellman value function to construct the Hamiltonian.

Theorem 8 (Hamilton-Jacobi-Bellman Equation). If the Bellman value function is twice differentiable, then we have

$$
\frac{\partial \mathcal{V}}{\partial t}(x, t)=\mathcal{H}\left(-\frac{\partial \mathcal{V}}{\partial q}(x, t), x, t\right)
$$

Moreover,

$$
p_{*}(t)=-\frac{\partial \mathcal{V}}{\partial q}\left(q_{*}\right)(t) \quad \text { and } \quad \mathcal{H}\left(p_{*}, q_{*}\right)(t)=\frac{\partial \mathcal{V}}{\partial t}\left(q_{*}\right)(t)
$$

Proof. Using the Bellman principle of optimality (20), subtracting $\mathcal{V}(x, t)$, dividing by $\tau-t$, and taking the limit as $\tau$ approaches $t$ yields

$$
0=\underset{q: q(t)=x}{\operatorname{opt}}\left[\int_{t}^{\tau} \mathcal{L}(q, \dot{q})(s) d s+\mathcal{V}(q)(\tau)-\mathcal{V}(q)(t)\right]
$$




$$
\begin{aligned}
& =\underset{q: q(t)=x}{\operatorname{opt}}\left[\frac{1}{\tau-t} \int_{t}^{\tau} \mathcal{L}(q, \dot{q})(s) d s+\frac{\mathcal{V}(q)(\tau)-\mathcal{V}(q)(t)}{\tau-t}\right] \\
& =\operatorname{opt}_{q: q(t)=x}\left[\mathcal{L}(q, \dot{q})(t)+\frac{d}{d t} \mathcal{V}(q)(t)\right] \\
& =\operatorname{opt}_{q: q(t)=x}\left[\mathcal{L}(q, \dot{q})(t)+\frac{\partial \mathcal{V}}{\partial q}(q)(t) \cdot \dot{q}(t)+\frac{\partial \mathcal{V}}{\partial t}(q)(t)\right] \\
& =\underset{v \in \mathbb{R}}{\operatorname{opt}}\left[\mathcal{L}(x, v, t)+\frac{\partial \mathcal{V}}{\partial q}(x, t) \cdot v+\frac{\partial \mathcal{V}}{\partial t}(x, t)\right] \\
& =\operatorname{opt}_{v \in \mathbb{R}}\left[\mathcal{L}(x, v, t)+\frac{\partial \mathcal{V}}{\partial q}(x, t) \cdot v\right]+\frac{\partial \mathcal{V}}{\partial t}(x, t) .
\end{aligned}
$$

So finally, we have

$$
\frac{\partial \mathcal{V}}{\partial t}(x, t)=-\operatorname{opt}_{v \in \mathbb{R}}\left[\mathcal{L}(x, v, t)+\frac{\partial \mathcal{V}}{\partial q}(x, t) \cdot v\right]=\underset{v \in \mathbb{R}}{\mathrm{opt}}\left[-\frac{\partial \mathcal{V}}{\partial q}(x, t) \cdot v-\mathcal{L}(x, v, t)\right],
$$

which yields (21). Finally, (22) follows from using the Bellman principle of optimality. For every intermediate time $\tau$, where $0 \leq \tau \leq T$, we have an equivalent optimization problem where the Bellman value function is now the additional terminal value function at the new terminal time $\tau$. Using (3) from Corollary 1 yields, on the left-hand side, the formula for the generalized momentum variable $p_{*}(t)$. From this follows the right-hand side formula of (3) for the Hamiltonian.

For operations research problems, the Bellman value function formulation is useful from a perspective of interpreting optimality. The partial derivatives have the interpretation of opportunity costs. The Hamilton-Jacobi-Bellman equation says that the generalized momentum variable is the opportunity cost density and the Hamiltonian is the opportunity cost rate.

\section{Optimal Control}

In this section we show how all three methods of dynamic optimizations, the Lagrangian, Hamiltonian, and Bellman value function, all contribute to solving problems in optimal control.

$$
\widehat{\mathcal{V}}(x, t) \equiv \operatorname{opt}_{\substack{q: q(t)=x, r \in K \\ \dot{q}=\hat{l}(q(t), r(t), t)}}\left[\int_{t}^{T} \hat{\mathcal{L}}(q, r)(s) d s\right],
$$

where $K$ is some compact subset of $\mathbb{R}$. Moreover, $\hat{\mathcal{L}}: \mathbb{R} \times K \times[0, T] \rightarrow \mathbb{R}$ is differentiable in the first and last arguments but optimally semi-continuous (i.e., upper semi-continuous when opt is max and lower semi-continuous otherwise) with respect to the middle argument. We define $\hat{l}: \mathbb{R} \times K \times[0, T] \rightarrow \mathbb{R}$ in a similar manner except that we assume that it is a continuous function of the middle argument.

Since this optimization problem only involves the state variable $r$ and not its time derivative, we refer to $r$ as a control variable. We want to extend our methods for dynamic optimization to cover the cases where the control variable range is confined to a compact set and the Lagrangian may not be a differentiable function in the control variable argument.

\subsection{Pontryagin Principle}

Suppose that we are given the control variable $r$. Our problem is then one of constrained optimization. The Lagrangian $\mathcal{L}$ for this problem is

$$
\mathcal{L}(p, q, \dot{q}, r, t)=\hat{\mathcal{L}}(q, r, t)+p \cdot(\dot{q}-\hat{l}(q, r, t)) .
$$


Since this Lagrangian is a linear function of $\dot{q}$, its Legendre transform is well defined exactly at $p$, which yields

$$
\mathcal{H}(p, q, r, t)=p \cdot \hat{l}(q, r, t)-\hat{\mathcal{L}}(q, r, t) .
$$

In this section we show that $\widehat{\mathcal{V}}(x, t)$ is a Bellman value function by deriving its Hamiltonian. We achieve this by constructing the position and momentum variables that solve Hamilton's equations.

Theorem 9 (Pontryagin Principle). For this optimal control problem, we have

$$
\mathcal{H}\left(p_{*}, q_{*}, r_{*}\right)(t)=\overline{\rho \in K} \underset{\mathrm{opt}}{\mathcal{H}}\left(p_{*}(t), q_{*}(t), \rho, t\right),
$$

where

$$
\dot{p}_{*}(t)=-\frac{\partial \mathcal{H}}{\partial q}\left(p_{*}, q_{*}, r_{*}\right)(t) \quad \text { and } \quad \dot{q}_{*}(t)=\frac{\partial \mathcal{H}}{\partial p}\left(p_{*}, q_{*}, r_{*}\right)(t)
$$

for all $0 \leq t \leq T$ with $p_{*}(T)=0$, and $q_{*}(0)$ is given .

Proof. Given a optimal path $q_{*}:[0, T] \rightarrow \mathbb{R}$ and an optimal control $r_{*}:[0, T] \rightarrow K$, we need only show that

$$
p_{*}(t)=-\frac{\partial \widehat{\mathcal{V}}}{\partial q}\left(q_{*}\right)(t) .
$$

Since we have

$$
\widehat{\mathcal{V}}\left(q_{*}\right)(t) \equiv \int_{t}^{T} \hat{\mathcal{L}}\left(q_{*}, r_{*}\right)(s) d s
$$

it follows that

$$
\frac{\partial \widehat{\mathcal{V}}}{\partial t}\left(q_{*}\right)(t)=-\frac{\partial \widehat{\mathcal{V}}}{\partial q}\left(q_{*}\right)(t) \cdot \hat{l}\left(q_{*}, r_{*}\right)(t)-\hat{\mathcal{L}}\left(q_{*}, r_{*}\right)(t)
$$

Equating this with (23) of Lemma 2, when $x=q_{*}(t)$, yields

$$
-\frac{\partial \widehat{\mathcal{V}}}{\partial q}\left(q_{*}\right)(t) \cdot \hat{l}\left(q_{*}, r_{*}\right)(t)-\hat{\mathcal{L}}\left(q_{*}, r_{*}\right)(t)=\overline{\mathrm{opt}}\left[-\frac{\partial \widehat{\mathcal{V}}}{\partial q}\left(q_{*}\right)(t) \cdot \hat{l}\left(q_{*}(t), \rho, t\right)-\hat{\mathcal{L}}\left(q_{*}(t), \rho, t\right)\right] .
$$

Finally, we use (25) with $x=q_{*}(t), \rho_{*}(x, t)=r_{*}(t)$ and the chain rule to show that

$$
\frac{d}{d t}\left(-\frac{\partial \widehat{\mathcal{V}}}{\partial q}\left(q_{*}\right)(t)\right)=-\left(-\frac{\partial \widehat{\mathcal{V}}}{\partial q}\left(q_{*}\right)(t) \cdot \frac{\partial \hat{l}}{\partial q}\left(q_{*}, r_{*}\right)(t)-\frac{\partial \hat{\mathcal{L}}}{\partial q}\left(q_{*}, r_{*}\right)(t)\right)
$$

Lemma 2. We have for all $x$ and $t$

$$
\frac{\partial \widehat{\mathcal{V}}}{\partial t}(x, t)=\underset{\rho \in K}{\operatorname{opt}} \mathcal{H}\left(-\frac{\partial \widehat{\mathcal{V}}}{\partial q}(x, t), x, \rho, t\right)
$$

where there exists some $\rho_{*}(x, t) \in K$ such that

$$
\frac{\partial \widehat{\mathcal{V}}}{\partial t}(x, t)=\mathcal{H}\left(-\frac{\partial \widehat{\mathcal{V}}}{\partial q}(x, t), x, \rho_{*}(x, t), t\right)
$$

and

$$
\frac{\partial^{2} \widehat{\mathcal{V}}}{\partial t \partial q}(x, t)+\frac{\partial^{2} \widehat{\mathcal{V}}}{\partial q^{2}}(x, t) \cdot \hat{l}\left(x, \rho_{*}(x, t), t\right)=\frac{\partial \mathcal{H}}{\partial q}\left(-\frac{\partial \widehat{\mathcal{V}}}{\partial q}(x, t), x, \rho_{*}(x, t), t\right) .
$$


Proof. Using a similar argument to the one we use for the Bellman value function, we have

$$
\begin{aligned}
& 0=\underset{q: q(t)=x}{\operatorname{opt}}\left[\int_{t}^{\tau} \hat{\mathcal{L}}(q, r)(s) d s+\widehat{\mathcal{V}}(q)(\tau)-\widehat{\mathcal{V}}(q)(t)\right] \\
& \dot{q}=\hat{l}(q, r)(t) \\
& =\operatorname{opt}_{\substack{q(t)=x \\
q=(q, r)(t)}}\left[\frac{1}{\tau-t} \int_{t}^{\tau} \hat{\mathcal{L}}(q, r)(s) d s+\frac{\widehat{\mathcal{V}}(q)(\tau)-\widehat{\mathcal{V}}(q)(t)}{\tau-t}\right] \\
& \dot{q}=\hat{l}(q, r)(t) \\
& =\underset{q: q(t)=x, r \in K}{\operatorname{opt}}\left[\hat{\mathcal{L}}(q, r)(t)+\frac{d}{d t} \widehat{\mathcal{V}}(q)(t)\right] \\
& \dot{q}=\hat{l}(q, r)(t) \\
& =\underset{q: q(t)=x, r \in K}{\operatorname{opt}}\left[\hat{\mathcal{L}}(q, r)(t)+\frac{\partial \widehat{\mathcal{V}}}{\partial q}(q)(t) \cdot \dot{q}(t)+\frac{\partial \widehat{\mathcal{V}}}{\partial t}(q)(t)\right] \\
& \dot{q}=\hat{l}(q, r)(t) \\
& =\underset{q: q(t)=x, r \in K}{\operatorname{opt}}\left[\hat{\mathcal{L}}(q, r)(t)+\frac{\partial \widehat{\mathcal{V}}}{\partial q}(q)(t) \cdot \hat{l}(q, r)(t)+\frac{\partial \widehat{\mathcal{V}}}{\partial t}(q)(t)\right] \\
& \dot{q}=\hat{l}(q, r)(t) \\
& =\underset{\rho \in K}{\operatorname{opt}}\left[\hat{\mathcal{L}}(x, \rho, t)+\frac{\partial \widehat{\mathcal{V}}}{\partial q}(x, t) \cdot \hat{l}(x, \rho, t)+\frac{\partial \widehat{\mathcal{V}}}{\partial t}(x, t)\right] \\
& =\underset{\rho \in K}{\operatorname{opt}}\left[\hat{\mathcal{L}}(x, \rho, t)+\frac{\partial \widehat{\mathcal{V}}}{\partial q}(x, t) \cdot \hat{l}(x, \rho, t)\right]+\frac{\partial \widehat{\mathcal{V}}}{\partial t}(x, t) \text {. }
\end{aligned}
$$

Since opt $(-x,-y)=-\overline{\operatorname{opt}}(x, y)$, we then have

$$
\frac{\partial \widehat{\mathcal{V}}}{\partial t}(x, t)=\underset{\rho \in K}{\mathrm{opt}}\left[-\frac{\partial \widehat{\mathcal{V}}}{\partial q}(x, t) \cdot \hat{l}(x, \rho, t)-\hat{\mathcal{L}}(x, \rho, t)\right] .
$$

An optimum semi-continuous function always attains its optimum on a compact set. Hence, there exists a $\rho_{*}(x, t)$ such that

$$
\frac{\partial \widehat{\mathcal{V}}}{\partial t}(x, t)=-\frac{\partial \widehat{\mathcal{V}}}{\partial q}(x, t) \cdot \hat{l}\left(x, \rho_{*}(x, t), t\right)-\hat{\mathcal{L}}\left(x, \rho_{*}(x, t), t\right) .
$$

Applying a weak version of the envelope lemma, see Lemma 3, we can then take the partial with respect to $x$ in the $q$ entry and obtain (25).

\subsection{Weak Envelope Lemma}

Now we state and prove the weak envelope lemma.

Lemma 3 (Weak Envelope Lemma). Let $f: \mathcal{O} \times K \rightarrow \mathbb{R}$ be differentiable in the first variable and optimally semi-continuous in the second where $\mathcal{O}$ and $K$ are, respectively, open and compact subsets of $\mathbb{R}$. If there exists some mapping $\phi: \mathbb{R} \rightarrow K$ such that

$$
f(x, \phi(x))=\underset{y \in K}{\operatorname{opt}} f(x, y),
$$

then

$$
\frac{d}{d x} f(x, \phi(x))=0 \quad \text { for all } x \in \mathcal{O} \text { implies that } \frac{\partial f}{\partial x}(x, \phi(x))=0 \quad \text { for all such } x .
$$

Proof. For all $x$ and $y$, we have

$$
f(x, \phi(x))=f(y, \phi(y)) \succeq_{\text {opt }} f(y, \phi(x)) .
$$

This means that $f(\cdot, \phi(x))$ has a local optimum at $x$, and so its partial derivative with respect to that argument is zero. 


\section{Optimizing Dynamical Queueing Systems}

In this section we combine dynamic rate queueing analysis and optimization to address three decision problems in operations management. These three examples analyze three distinct queuing systems: a loss system as well as both a single server and multiserver delay system. Provisioning, pricing, and prioritization problems are considered in the examples.

\subsection{Pricing Policy for a Loss System}

First, let us consider the following dynamic pricing problem for a loss system as illustrated in Figure 8. The service demand is a function of time and is sensitive to the price of the service. Upon arrival to the system, with fixed capacity $C$, a joining customer pays $\pi$ dollars. The service provider guarantees a blocking probability no larger than $\epsilon$. The service provider uses price as a dynamic control to maximize revenue while maintaining the blocking probability service guarantees. The modified offered load approximation (Jagerman [24]) leads to a deterministic version of the problem (see $\S 2.3$ ).

\section{Problem 5.}

$$
r(T)=\max _{\{\pi(t): 0 \leq t \leq T\}} \int_{0}^{T} \pi(t) \lambda(t, \pi(t)) d t
$$

subject to

$$
\dot{q}(t)=\lambda(t, \pi)-\mu \cdot q(t) \quad \text { where } q(t) \leq \theta .
$$

Recall that $\theta$ is the threshold offered load. This is defined by setting $\beta_{C}(\theta)=\epsilon$, where $\beta_{C}$ is the Erlang blocking formula.

We begin the analysis by writing down the Lagrangian for the problem. To do this, we introduce a slack variable for the inequality constraint. The pricing decision for this Erlang loss system is made by optimizing the integral of the Lagrangian

$$
\mathcal{L}(p, \pi, q, \dot{q}, x, y)=\pi \cdot \lambda(\pi)+p \cdot(\dot{q}-\lambda(\pi)+\mu \cdot q)+x \cdot\left(\theta-q-y^{2}\right),
$$

where $y$ is a slack variable. From the Euler-Lagrange equations, we obtain

$$
0=\frac{d}{d t} \frac{\partial \mathcal{L}}{\partial \dot{x}}=\frac{\partial \mathcal{L}}{\partial x} \Longleftrightarrow \theta-q_{*}=y_{*}^{2} \quad \text { or } \quad \theta \geq q_{*}
$$

and

$$
0=\frac{d}{d t} \frac{\partial \mathcal{L}}{\partial \dot{y}}=\frac{\partial \mathcal{L}}{\partial y} \Longleftrightarrow x_{*} \cdot y_{*}=0 \quad \text { or } \quad x_{*} \cdot\left(\theta-q_{*}\right)=0
$$

FiguRE 8. Diagram of multiserver loss queueing model for pricing decision.

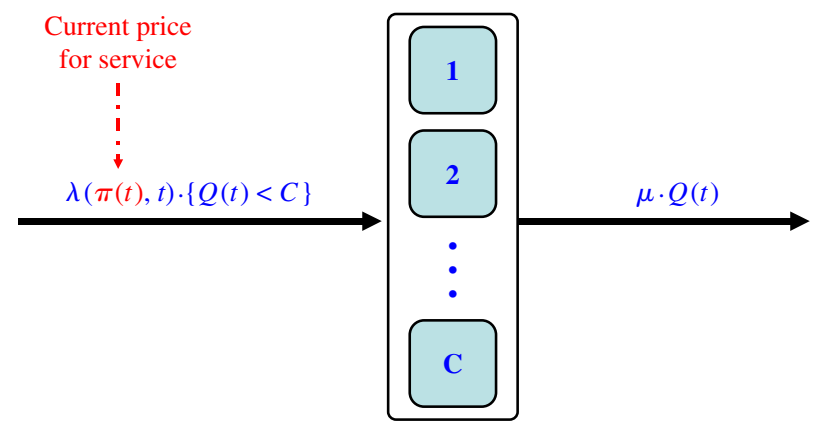


Using sensitivity analysis, we have

$$
\frac{d}{d \theta}\left(\pi_{*} \cdot \lambda\left(\pi_{*}\right)\right)=x_{*}
$$

The Hamiltonian for this system is

$$
\mathcal{H}(p, \pi, q, x, y)=(p-\pi) \cdot \lambda(\pi)+x \cdot\left(y^{2}+q-\theta\right)-\mu \cdot p \cdot q .
$$

Hence by using Hamilton's equations, we obtain the dynamical system formed by

$$
\dot{p}=-\frac{\partial \mathcal{H}}{\partial q} \Longleftrightarrow \dot{p}_{*}=\mu \cdot p_{*}-x_{*}
$$

and

$$
\dot{q}=\frac{\partial \mathcal{H}}{\partial p} \Longleftrightarrow \dot{q}_{*}=\lambda\left(\pi_{*}\right)-\mu \cdot q_{*}
$$

Applying the Pontryagin principle yields

$$
x_{*} \cdot y_{*}^{2}=\min _{z \in \mathbb{R}} x_{*} \cdot z^{2}=0 \Longleftrightarrow x_{*} \geq 0
$$

and

$$
\left(p_{*}(t)-\pi_{*}(t)\right) \cdot \lambda\left(\pi_{*}\right)(t)=\min _{z \in \mathbb{R}}\left(p_{*}(t)-z\right) \cdot \lambda(z, t) .
$$

\subsection{Provisioning Policy for a Multiserver Delay System}

Motivated by call center staffing, we examine the dynamic provisioning of resources in a multiserver queue with $C$ servers as illustrated in Figure 9. Let customers arrive according to a nonhomogeneous Poisson process. Further, let the service times be exponentially distributed. Upon completion a customers pays the system $r$ dollars. The cost per unit time for resources is an increasing concave function of the number of servers, $w(C)$, where $w(0)=0$. The service provider's objective is to maximize the amount of profit generated by dynamically selecting the number of servers over a finite period of time. Following $§ 2.4$, the number of customers in the system is modeled by a multiserver queue with nonhomogeneous Poisson arrivals with infinite capacity waiting space and a FCFS customer service discipline. Using Markovian service networks (Mandelbaum et al. [33]) and strong approximation theory (see $\S 2.4$ ) we have a deterministic approximate problem.

Problem 6.

$$
r(T)=\max _{\{C(t): 0 \leq t \leq T\}} \int_{0}^{T} r \mu \cdot \min (q(t), C(t))-w(C(t)) d t
$$

where

$$
\dot{q}(t)=\lambda(t)-\min (q(t), C(t)) .
$$

The provisioning decision for this multiserver delay system is made by optimizing the integral of the Lagrangian

$$
\mathcal{L}(C, p, q, \dot{q})=r \mu \cdot(q \wedge C)-w(C)+p \cdot(\dot{q}-\lambda+\mu \cdot(q \wedge C))
$$

If we construct the Hamiltonian, we obtain

$$
\mathcal{H}(C, p, q)=p \cdot(\lambda-\mu \cdot(q \wedge C))+w(C)-r \mu \cdot(q \wedge C) .
$$


FIGURE 9. Diagram of multiserver delay queueing model for provisioning decision.

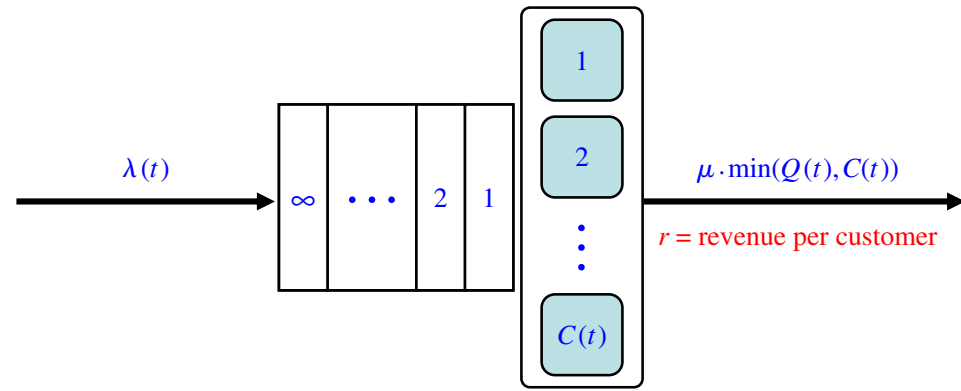

Now we make the assumption of economies of scale for the cost of the servers. This makes $w$ an increasing concave function and we then have by Pontryagin's principle

$$
\mathcal{H}\left(C_{*}, p_{*}, q_{*}\right)=\min _{C \geq 0} \mathcal{H}\left(C, p_{*}, q_{*}\right)=\mathcal{H}_{1}\left(p_{*}, q_{*}\right) \wedge \mathcal{H}_{2}\left(p_{*}, q_{*}\right),
$$

where

$$
\mathcal{H}_{1}(p, q) \equiv \mathcal{H}(0, p, q)=p \cdot \lambda
$$

and

$$
\mathcal{H}_{2}(p, q) \equiv \mathcal{H}(q, p, q)=p \cdot(\lambda-\mu \cdot q)+w(q)-r \mu \cdot q .
$$

Using Hamilton's equations, we then have

$$
\dot{p}_{*}= \begin{cases}0 & \text { when } \mathcal{H}_{1}\left(p_{*}, q_{*}\right)<\mathcal{H}_{2}\left(p_{*}, q_{*}\right), \\ \mu \cdot\left(p_{*}+r\right)-w^{\prime}\left(q_{*}\right) & \text { when } \mathcal{H}_{2}\left(p_{*}, q_{*}\right)<\mathcal{H}_{1}\left(p_{*}, q_{*}\right),\end{cases}
$$

where $p(T)=0$, and

$$
\dot{q}_{*}= \begin{cases}\lambda & \text { when } \mathcal{H}_{1}\left(p_{*}, q_{*}\right)<\mathcal{H}_{2}\left(p_{*}, q_{*}\right), \\ \lambda-\mu \cdot q_{*} & \text { when } \mathcal{H}_{2}\left(p_{*}, q_{*}\right)<\mathcal{H}_{1}\left(p_{*}, q_{*}\right),\end{cases}
$$

where $q(0)$ is given. We can then compute $p_{*}$ and $q_{*}$ by solving these equations. We can then determine $C_{*}$ to be

$$
C_{*}= \begin{cases}0 & \text { when } \mathcal{H}_{1}\left(p_{*}, q_{*}\right)<\mathcal{H}_{2}\left(p_{*}, q_{*}\right), \\ q_{*} & \text { when } \mathcal{H}_{2}\left(p_{*}, q_{*}\right)<\mathcal{H}_{1}\left(p_{*}, q_{*}\right) .\end{cases}
$$

\subsection{Prioritization Policy for a Single Server Delay System}

Next, we consider a priority problem for a multiclass PS queue. The service provider dynamically prioritizes each class to maximize revenue over a finite time horizon. The fluid model for the PS system with one permanent job and two customer classes is

$$
\dot{q}^{(i)}=\lambda_{i}-\theta^{(i)}(\mathbf{w}, \mathbf{q})
$$

with

$$
\theta^{(i)}(\mathbf{w}, \mathbf{q}) \equiv \frac{\mu_{1} w^{(i)} q^{(i)}}{w_{0}+w^{(1)} q^{(1)}+w^{(2)} q^{(2)}},
$$

for $i=1,2$. 
Using vector notation, we can write this as

$$
\dot{\mathbf{q}}=\boldsymbol{\lambda}-\boldsymbol{\theta}(\mathbf{w}, \mathbf{q}),
$$

where

and

$$
\mathbf{q}(t)=\left(q^{(1)}(t), q^{(2)}(t)\right), \quad \boldsymbol{\lambda}=\left(\lambda_{1}(t), \lambda_{2}(t)\right), \quad \mathbf{w}=\left(w^{(1)}, w^{(2)}\right)
$$

$$
\boldsymbol{\theta}(\mathbf{w}, \mathbf{q})=\left(\theta^{(1)}(\mathbf{w}, \mathbf{q}), \theta^{(2)}(\mathbf{w}, \mathbf{q})\right)
$$

where

$$
\theta^{(1)}(\mathbf{w}, \mathbf{q})=\frac{\mu_{1} w^{(1)} q^{(1)}}{w_{0}+w^{(1)} q^{(1)}+w^{(2)} q^{(2)}} \quad \text { and } \quad \theta^{(2)}(\mathbf{w}, \mathbf{q})=\frac{\mu_{2} w^{(2)} q^{(2)}}{w_{0}+w^{(1)} q^{(1)}+w^{(2)} q^{(2)}} .
$$

To maximize the revenue over time $(0, T]$, the Lagrangian is

$$
\mathcal{L}(\mathbf{p}, \mathbf{q}, \dot{\mathbf{q}}, \mathbf{w})=\mathbf{r} \cdot \boldsymbol{\theta}(\mathbf{w}, \mathbf{q})+\mathbf{p} \cdot(\dot{\mathbf{q}}-\boldsymbol{\lambda}+\boldsymbol{\theta}(\mathbf{w}, \mathbf{q})) .
$$

where $\mathbf{r}=\left(r_{1}, r_{2}\right)$ and $\mathbf{p}(t)=\left(p^{(1)}(t), p^{(2)}(t)\right)$. It follows that the Hamiltonian is

$$
\mathcal{H}(\mathbf{p}, \mathbf{q}, \mathbf{w})=\mathbf{p} \cdot \boldsymbol{\lambda}-(\mathbf{p}+\mathbf{r}) \cdot \boldsymbol{\theta}(\mathbf{w}, \mathbf{q}) .
$$

Now we define the total output rate at time $t$ when weighted completely for the class $i$ customer class and the permanent job or

$$
\hat{\theta}^{(i)}(t) \equiv \theta^{(i)}\left(\left(1-w_{0}\right) \cdot \mathbf{e}_{i}, \mathbf{q}(t)\right)=\frac{\mu_{i} \cdot\left(1-w_{0}\right) q^{(i)}(t)}{w_{0}+\left(1-w_{0}\right) q^{(i)}(t)} .
$$

Note that

$$
\lim _{w_{0} \downarrow 0} \hat{\theta}^{(i)}(t)= \begin{cases}\mu_{i} & \text { if } q^{(i)}(t)>0, \\ 0 & \text { if } q^{(i)}(t)=0 .\end{cases}
$$

Given the optimal phase-space path $\left(p_{*}, q_{*}\right)$, the weights $w^{(1)}$ and $w^{(2)}$ solve the following static optimization problem by Pontryagin's theorem.

$$
\max _{w: w_{0}+w^{(1)}+w^{(2)}=1, w^{(1)} \cdot w^{(2)} \geq 0} \mathcal{H}\left(\mathbf{p}_{*}, \mathbf{q}_{*}, \mathbf{w}\right)=\mathcal{H}_{1}\left(\mathbf{p}_{*}, \mathbf{q}_{*}\right) \wedge \mathcal{H}_{2}\left(\mathbf{p}_{*}, \mathbf{q}_{*}\right),
$$

where

$$
\mathcal{H}_{i}(\mathbf{p}, \mathbf{q}) \equiv \mathcal{H}\left(\mathbf{p}, \mathbf{q},\left(1-w_{0}\right) \cdot \mathbf{e}_{i}\right)=p^{(1)} \lambda_{1}+p^{(2)} \lambda_{2}-\left(p^{(i)}+r_{i}\right) \cdot \hat{\theta}^{(i)}
$$

Hence, the index $i$ yields the smallest opportunity cost rate $\mathcal{H}_{i}(\mathbf{p}, \mathbf{q})$, or equivalently the largest value rate of the competing Lagrangians, if and only if it yields the largest $\left(p^{(i)}+r_{i}\right)$. $\hat{\theta}^{(i)}$. This product is a variation on the $c \mu$-rule. Here, the total "reward" per class $i$ customer is $p^{(i)}+r_{i}$, which is the revenue from serving a class $i$ customer plus the opportunity cost savings obtained from having a customer depart the system now.

Finally, when $i$ indexes the smallest Hamiltonian (or the largest Lagrangian), then from Hamilton's equations we have

$$
\begin{aligned}
\dot{p}_{*}^{(i)} & =-\frac{\partial}{\partial q^{(i)}} \mathcal{H}_{i}\left(\mathbf{p}_{*}, \mathbf{q}_{*}, \mathbf{w}_{*}\right) \\
& =\left.\left(p_{*}^{(i)}+r_{i}\right) \cdot \mu_{i} \cdot \frac{\partial}{\partial q^{(i)}}\left(1-\frac{w_{0}}{w_{0}+\left(1-w_{0}\right) q^{(i)}}\right)\right|_{q^{(i)}=q_{*}^{(i)}} \\
& =\frac{\left(p_{*}^{(i)}+r_{i}\right) \cdot \mu_{i} w_{0} \cdot\left(1-w_{0}\right)}{\left(w_{0}+\left(1-w_{0}\right) q_{*}^{(i)}\right)^{2}} \\
& =\left(p_{*}^{(i)}+r_{i}\right) \cdot \hat{\theta}_{*}^{(i)} \cdot\left(1-\frac{\hat{\theta}_{*}^{(i)}}{\mu_{i}}\right) .
\end{aligned}
$$

Otherwise, we have $\dot{p}_{*}^{(i)}=0$. We either have $\dot{q}_{*}^{(i)}=\lambda_{i}-\hat{\theta}_{*}^{(i)}$ or $\dot{q}_{*}^{(i)}=\lambda_{i}$, respectively. 
To maximize the revenue over time $(0, T]$ with a fair allocation constraint, the Lagrangian is now

$$
\begin{aligned}
\mathcal{L}(\mathbf{p}, \mathbf{q}, \dot{\mathbf{q}}, x, \dot{y}, \mathbf{w})= & \mathbf{r} \cdot \boldsymbol{\theta}(\mathbf{w}, \mathbf{q})+\mathbf{p} \cdot(\dot{\mathbf{q}}-\boldsymbol{\lambda}+\boldsymbol{\theta}(\mathbf{w}, \mathbf{q})) \\
& +x \cdot\left(\dot{y}-\delta_{1} \cdot \theta^{(2)}(\mathbf{w}, \mathbf{q})+\delta_{2} \cdot \theta^{(1)}(\mathbf{w}, \mathbf{q})\right),
\end{aligned}
$$

where $y(0)=y(T)=0$. Since the Lagrangian has no explicit dependence on $y$, then this makes the multiplier (or dual) variable $x$ a constant. Our Hamiltonian is now

$$
\mathcal{H}(\mathbf{p}, \mathbf{q}, x, \mathbf{w})=\mathbf{p} \cdot \boldsymbol{\lambda}-(\mathbf{p}+\mathbf{r}) \cdot \boldsymbol{\theta}(\mathbf{w}, \mathbf{q})+x \cdot\left(\delta_{1} \cdot \theta^{(2)}(\mathbf{w}, \mathbf{q})-\delta_{2} \cdot \theta^{(1)}(\mathbf{w}, \mathbf{q})\right) .
$$

Using similar arguments to the unconstrained case, we have

$$
\max _{w: w_{0}+w^{(1)}+w^{(2)}=1, w^{(1)} \cdot w^{(2)} \geq 0} \mathcal{H}\left(\mathbf{p}_{*}, \mathbf{q}_{*}, \mathbf{w}, x\right)=\mathcal{H}_{1}\left(\mathbf{p}_{*}, \mathbf{q}_{*}, x\right) \wedge \mathcal{H}_{2}\left(\mathbf{p}_{*}, \mathbf{q}_{*}, x\right),
$$

where

$$
\mathcal{H}_{1}(\mathbf{p}, \mathbf{q}, x) \equiv \mathcal{H}\left(\mathbf{p}, \mathbf{q},\left(1-w_{0}\right) \cdot \mathbf{e}_{1}, x\right)=p^{(1)} \lambda_{1}+p^{(2)} \lambda_{2}-\left(p^{(1)}+r_{1}+\delta_{2} \cdot x\right) \cdot \hat{\theta}^{(1)}
$$

and

$$
\mathcal{H}_{2}(\mathbf{p}, \mathbf{q}, x) \equiv \mathcal{H}\left(\mathbf{p}, \mathbf{q},\left(1-w_{0}\right) \cdot \mathbf{e}_{2}, x\right)=p^{(1)} \lambda_{1}+p^{(2)} \lambda_{2}-\left(p^{(1)}+r_{1}-\delta_{1} \cdot x\right) \cdot \hat{\theta}^{(2)} .
$$

\section{Summary}

Using the historical communications motivations for queueing theory such as building the telephone network and designing the Internet, we can organize our fundamental queueing models as follows:

1. service demand (arrival traffic);

2. resource demand (offered load);

3. service loss (voice services);

4. service delay (data services).

From an operations research perspective, we use the Lagrangian formulation to model the profit, revenue, and cost rates for our dynamic optimization problems. Through the use of auxiliary variables, we can incorporate equality, inequality and isoperimetric constraints into our dynamic optimization model. For example, a manager may want to achieve profit maximization within the constraint of some acceptable quality of service level. Below, we summarize the types of variables that we use with Lagrangians:

1. state variables;

2. multiplier variables;

3. dual variables;

4. slack variables;

5. control variables;

6. constants.

The main use of the Hamiltonian formulation here is computational. Unlike the Euler-Lagrange equations, Hamilton's equations are equivalent but always form an evendimensional dynamical system. Moreover, given the value of the state and dual variables, the corresponding control variable solves a static optimization problem for the Hamiltonian. Unfortunately, we have the mixed boundary conditions of the initial state variable and dual state variable values. Specific examples, physical intuition, and numerical methods suggest simple iterative methods that can find the solution to these equations. 
In the framework of classical mechanics, the Lagrangian does not appear to have a physical interpretation. It is merely the mathematical object whose optimized time-integral yields Newtonian mechanics. By contrast, the Hamiltonian and the momentum variable have physical interpretations since they can be conserved quantities. Care must be taken to numerically integrate the time evolution of the state and dual variables in time directions that respect the well posedness of their governing differential equation.

In the framework of queueing operations, unlike physics, the Lagrangian has a natural interpretation as a value rate. Instead of saying "kinetic minus potential energies," we can say "revenue minus cost rates," and this is a "profit rate." At first glance, the Hamiltonian and the dual or generalized momentum variables do not seem to have natural interpretations, which is the reverse of situation in physics. We can remedy this situation, however, by using the Bellman value function, the Hamilton-Jacobi-Bellman equations and the economic interpretation of the partial derivative. If our state variable is the average number of customers in the queueing system, then our momentum variable is the opportunity cost per customer incurred by admitting a customer. In general, it can be viewed as an opportunity cost density. The Hamiltonian is now the opportunity cost rate since it is the partial time derivative of the Bellman value function. Table 1 summarizes these relationships between the operational queueing quantities of interest and their classical mechanical counterparts.

The four fundamental operations problems that we address using these dynamical systems and dynamic optimization methods are as follows:

1. performance (quality of service predictions);

2. pricing (quality of service control policies);

3. prioritization (resource allocation policies);

4. provisioning (resource design policies).

By performance, we mean using queueing theory to make quality of service predictions for the communications system. The price of service influences its demand; hence dynamic pricing can control the quality of service. The pricing policy from our analysis of a multiserver loss queue can be summarized in Table 2. Given a fixed number of resources, prioritization policies efficiently allocate scarce resources to the differing classes of customers. The prioritization policy from our analysis of a single server delay queue can be summarized in Table 3. Finally, provisioning policies enable the manager to design a communication service by finding the most profit optimal way to add resources over time. The provisioning policy from our analysis of a multiserver delay queue can be summarized in Table 4.

It is through the analysis of various fundamental dynamic rate queues that we find simple, low-dimensional, dynamical systems to approximate the time evolution of the mean behavior of the original queueing model. In this tutorial, we show that calculus of variations techniques can give insight into the canonical dynamic optimization issues of dynamic rate queues motivated by communication decision problems.

TABLE 1. Classical mechanical terms and their queueing operations counterparts.

\begin{tabular}{lcc}
\hline & Mechanics & Operations \\
\hline$q$ & Position & Number of customers in service \\
$\dot{q}$ & Velocity & Customer flow rate \\
$\mathcal{L}(q, \dot{q})$ & Lagrangian & Value rate \\
$p$ & Momentum & Opportunity cost per customer \\
$\mathcal{H}(p, q)$ & Hamiltonian & Opportunity cost rate \\
$\mathcal{V}(q)$ & Action & Bellman value function \\
\hline
\end{tabular}


TABLE 2. Pricing policy for a multiserver loss queue.

\begin{tabular}{|c|c|c|c|c|c|}
\hline $\begin{array}{l}\text { Time } \\
\text { region }\end{array}$ & $\begin{array}{c}\text { Dual } \\
\text { variable }\end{array}$ & $\begin{array}{c}\text { State } \\
\text { variable }\end{array}$ & $\begin{array}{l}\text { Control } \\
\text { variable }\end{array}$ & $\begin{array}{l}\text { Multiplier } \\
\text { variable }\end{array}$ & $\begin{array}{c}\text { Slack } \\
\text { variable }\end{array}$ \\
\hline$q_{*}<\theta$ & $\dot{p}_{*}=\mu \cdot p_{*}$ & $\dot{q}_{*}=\lambda\left(\pi_{*}\right)-\mu \cdot q_{*}$ & $\begin{aligned} \lambda\left(\pi_{*}\right)= & \left(p_{*}-\pi_{*}\right) \\
& \cdot \lambda^{\prime}\left(\pi_{*}\right)\end{aligned}$ & $x_{*}=0$ & $y_{*}^{2}=\theta-q_{*}$ \\
\hline$q_{*}=\theta$ & $\dot{p}_{*}=\mu \cdot p_{*}-x_{*}$ & $\dot{q}_{*}=-\left(\mu \cdot q_{*}-\lambda\left(\pi_{*}\right)\right)^{+}$ & $\pi_{*}=\lambda^{-1}(\mu \cdot \theta)$ & $\begin{array}{c}x_{*}=\mu \\
\cdot\left(\pi_{*}+1 / \lambda^{\prime}\left(\pi_{*}\right)\right)\end{array}$ & $y_{*}=0$ \\
\hline
\end{tabular}

TABLE 3. Prioritization policy for a single server delay queue.

\begin{tabular}{lccc}
\hline $\begin{array}{l}\text { Opportunity cost } \\
\text { rate minimum }\end{array}$ & $\begin{array}{c}\text { Dual } \\
\text { variable }\end{array}$ & $\begin{array}{c}\text { State } \\
\text { variable }\end{array}$ & $\begin{array}{c}\text { Control } \\
\text { variable }\end{array}$ \\
\hline $\mathcal{H}_{1}\left(\mathbf{p}_{*}, \mathbf{q}_{*}, \mathbf{w}_{*}\right)$ & $\dot{p}_{*}^{(1)}=\left(p_{*}^{(1)}+r_{1}\right) \cdot \hat{\theta}_{*}^{(1)}(t)$ & $\dot{q}_{*}^{(1)}=\lambda_{1}-\mu_{1} \cdot q_{*}^{(1)}$ & $w_{*}^{(1)}=1-w_{0}$ \\
& $\cdot\left(1-\hat{\theta}_{*}^{(1)}(t) / \mu_{1}\right)$ & $\dot{q}_{*}^{(2)}=\lambda_{2}$ & $w_{*}^{(2)}=0$ \\
& $\dot{p}_{*}^{(2)}=0$ & & \\
$\dot{p}_{*}^{(1)}=0$ & $\dot{q}_{*}^{(1)}=\lambda_{1}$ & $w_{*}^{(1)}=0$ \\
$\mathcal{H}_{2}\left(\mathbf{p}_{*}, \mathbf{q}_{*}, \mathbf{w}_{*}\right)$ & $\dot{p}_{*}^{(2)}=\left(p_{*}^{(2)}+r_{2}\right) \cdot \hat{\theta}_{*}^{(2)}(t)$ & $\dot{q}_{*}^{(2)}=\lambda_{2}-\mu_{2} \cdot q_{*}^{(2)}$ & $w_{*}^{(2)}=1-w_{0}$ \\
& $\cdot\left(1-\hat{\theta}_{*}^{(2)}(t) / \mu_{2}\right)$ & & \\
\hline
\end{tabular}

TABLE 4. Provisioning policy for a multiserver delay queue.

\begin{tabular}{lccr}
\hline $\begin{array}{l}\text { Opportunity cost } \\
\text { rate minimum }\end{array}$ & $\begin{array}{c}\text { Dual } \\
\text { variable }\end{array}$ & $\begin{array}{c}\text { State } \\
\text { variable }\end{array}$ & $\begin{array}{c}\text { Control } \\
\text { variable }\end{array}$ \\
\hline $\mathcal{H}_{1}\left(p_{*}, q_{*}\right)$ & $\dot{p}_{*}=0$ & $\dot{q}_{*}=\lambda$ & $C_{*}=0$ \\
$\mathcal{H}_{2}\left(p_{*}, q_{*}\right)$ & $\dot{p}_{*}=\mu \cdot\left(p_{*}+r\right)-w^{\prime}\left(q_{*}\right)$ & $\dot{q}_{*}=\lambda-\mu \cdot q_{*}$ & $C_{*}=q_{*}$ \\
\hline
\end{tabular}

\section{Appendix. Linear Dynamical Systems and Markov Processes}

\section{Matrix Exponentials}

Ordinary differential equations that only involve first derivatives are not as limiting as might first appear. Consider the following example.

Problem 7 (Linear Ordinary Differential Equations). Consider the differentiable function $x:[0, T] \rightarrow \mathbb{R}$ that solves the $n$th order ordinary differential equation

$$
a_{0} \cdot x(t)+a_{1} \cdot \frac{d x}{d t}(t)+\cdots+a_{n} \cdot \frac{d^{n} x}{d t^{n}}(t)=0 .
$$

Rewrite this equation as a linear function of $q$ and $\frac{d}{d t} q$.

Solution. If we define $\mathbf{q}(t)$, where

$$
\mathbf{q}(t)=\left[x(t), \frac{d x}{d t}(t), \ldots, \frac{d^{n-1} x}{d t^{n-1}}(t)\right],
$$

then we can rewrite (30) as

$$
\frac{d}{d t} \mathbf{q}(t)=\mathbf{q}(t) \cdot \mathbf{A}
$$


where

$$
\mathbf{A}=\left[\begin{array}{cccccc}
0 & 0 & 0 & \cdots & 0 & -a_{0} / a_{n} \\
1 & 0 & 0 & \cdots & 0 & -a_{1} / a_{n} \\
0 & 1 & 0 & \cdots & 0 & -a_{2} / a_{n} \\
\vdots & \ddots & \ddots & \ddots & \vdots & \vdots \\
0 & 0 & 0 & \ddots & 0 & -a_{n-2} / a_{n} \\
0 & 0 & 0 & \cdots & 1 & -a_{n-1} / a_{n}
\end{array}\right]
$$

The deterministic process $\{\mathbf{q}(t) \mid t \geq 0\}$ is referred to as a linear dynamical system. Moreover, it has the explicit solution of

$$
\mathbf{q}(t)=\mathbf{q}(0) \cdot \exp (t \mathbf{A}),
$$

where the square matrix factor is called the matrix exponential or

$$
\exp (t \mathbf{A})=\sum_{n=0}^{\infty} \frac{t^{n}}{n !} \mathbf{A}^{n} .
$$

It is important to note that if the coefficients of the ordinary differential equation (or the matrix $\mathbf{A}$ ) are time dependent, then the solution to $\mathbf{q}(t)$ is not the matrix exponential of the integral of the family of matrices $\{\mathbf{A}(s) \mid 0 \leq s \leq t\}$. This means that, in general, the solution to

$$
\frac{d}{d t} \mathbf{q}(t)=\mathbf{q}(t) \cdot \mathbf{A}(t)
$$

is not the matrix exponential of the integral of this family of matrices or

$$
\mathbf{q}(t) \neq \mathbf{q}(0) \cdot \exp \left(\int_{0}^{t} \mathbf{A}(s) d s\right)
$$

We can make this concept work, however, by using the convention of time-ordered operators due to Feynman [10]. First, define $\{\hat{\mathbf{A}}(s) \mid 0 \leq s \leq t\}$ to be a family of formal matrices with the property that for all pairs of times $r<s$, we have

$$
\hat{\mathbf{A}}(r) \cdot \hat{\mathbf{A}}(s)=\hat{\mathbf{A}}(s) \cdot \hat{\mathbf{A}}(r) \equiv \mathbf{A}(r) \cdot \mathbf{A}(s) .
$$

Now we can write our solution to (32), as the formal expression

$$
\mathbf{q}(t)=\mathbf{q}(0) \cdot \exp \left(\int_{0}^{t} \hat{\mathbf{A}}(s) d s\right) .
$$

\section{Uniformization and Uniform Acceleration}

For the case of a Markov process, the time evolution of the probability of being in a specific state is a dynamical system. These are also called the Kolmogorov forward equations. The governing matrix $\mathbf{A}$ is referred to as a Markov generator. Such generators have positive off-diagonal entries and row sums equal to zero. Assuming that all the diagonal terms over the interval $(0, t]$ are bounded by a constant $\alpha$, then we define the matrix $\mathbf{P}_{\alpha}(s)$ to be

$$
\mathbf{P}_{\alpha}(s) \equiv \mathbf{I}+\frac{1}{\alpha} \mathbf{A}(s)
$$


for all $0 \leq s \leq t$, where $\mathbf{I}$ is the identity matrix. These are called stochastic matrices where their entries are positive and row sums equal one. Using our operator convention and letting $\widehat{\mathbf{P}}_{\alpha}(t)$ be the time-ordered analogue to the stochastic matrix $\mathbf{P}_{\alpha}(t)$ at time $t$, we have

$$
\begin{aligned}
\exp \left(\int_{0}^{t} \hat{\mathbf{A}}(s) d s\right) & =\exp \left(-\alpha t \mathbf{I}+\int_{0}^{t} \alpha \mathbf{I}+\hat{\mathbf{A}}(s) d s\right) \\
& =e^{-\alpha t} \cdot \exp \left(\alpha t \cdot \frac{1}{t} \int_{0}^{t} \widehat{\mathbf{P}}_{\alpha}(s) d s\right) \\
& =\sum_{n=0}^{\infty} \frac{e^{-\alpha t} \cdot(\alpha t)^{n}}{n !}\left(\frac{1}{t} \int_{0}^{t} \widehat{\mathbf{P}}_{\alpha}(s) d s\right)^{n} \\
& =\sum_{n=0}^{\infty} \frac{e^{-\alpha t} \cdot(\alpha t)^{n}}{n !} \cdot \frac{n !}{t^{n}} \cdot \int_{0<s_{1}<\cdots<s_{n}<t} \widehat{\mathbf{P}}_{\alpha}\left(s_{1}\right) \cdots \widehat{\mathbf{P}}_{\alpha}\left(s_{n}\right) d s_{1} \cdots d s_{n} \\
& =\sum_{n=0}^{\infty} \frac{e^{-\alpha t} \cdot(\alpha t)^{n}}{n !} \cdot \int_{0<s_{1}<\cdots<s_{n}<t} \mathbf{P}_{\alpha}\left(s_{1}\right) \cdots \mathbf{P}_{\alpha}\left(s_{n}\right) \cdot \frac{n !}{t^{n}} d s_{1} \cdots d s_{n} .
\end{aligned}
$$

We now show that deeper results for Poisson processes give this sum a probabilistic interpretation.

The lead scalar factor for each summand is the probability of a Poisson process making $n$ jumps during the interval $(0, t]$. A homogeneous Poisson process has the property that conditioned on this event, the joint distribution of the $n$ jump times equals the same for the order statistics of $n$ i.i.d. random variables that are uniformly distributed on $(0, t]$. This means that the sample paths of this time-inhomogeneous Markov process are driven by the jumps of a Poisson process with rate $\alpha$. At precisely these jump times, the process makes an instantaneous transition according to a time-inhomogeneous Markov chains whose stochastic matrices are specified by the time of the Poisson jump.

In turn, this sample path decomposition, called uniformization, yields a sample path interpretation of uniform acceleration. For a given scale parameter $\eta>0$, we have

$$
\exp \left(\int_{0}^{t} \eta \cdot \hat{\mathbf{A}}(s) d s\right)=\sum_{n=0}^{\infty} \frac{e^{-\eta \alpha t} \cdot(\eta \alpha t)^{n}}{n !} \int_{0<s_{1}<\cdots<s_{n}<t} \ldots \int_{\alpha} \mathbf{P}_{\alpha}\left(s_{1}\right) \cdots \mathbf{P}_{\alpha}\left(s_{n}\right) \cdot \frac{n !}{t^{n}} d s_{1} \cdots d s_{n} .
$$

From this representation, we see that uniform acceleration speeds up the jump rate of the underlying Poisson process but does not change the stochastic matrices sampled at any of the jump times.

\section{References}

[1] C. E. Agnew. Dynamic modeling and control of congestion-prone systems. Operations Research 24 400-419, 1976.

[2] J. M. Alden and R. L. Smith. Rolling horizon procedures in nonhomogeneous Markov decision processes. Operations Research 40:183-194, 1992.

[3] J. C. Bean, W. J. Hopp, and I. Duenyas. A stopping rule for forecast horizons in nonhomogeneous Markov decision processes. Operations Research 40:1188-1199, 1992.

[4] R. Bellman. Dynamic Programming. Princeton University Press, Princeton, NJ, 1957.

[5] D. P. Bertsekas. Dynamic programming and optimal control. Athena Scientific. Belmont, MA, 2001.

[6] S. Eick, W. A. Massey, and W. Whitt. The physics of the $M(t) / G / \infty$ queue. Operations Research 41:400-408, 1993.

[7] A. K. Erlang. Solutions of some problems in the theory of probabilities of significance in automatic telephone exchanges. The Post Office Electrical Engineers' Journal 10:189-197, 1918. [Originally published in Danish. +Elektroteknikeren 13, 1917]. 
[8] S. N. Ethier and T. G. Kurtz. Markov Process: Characterization and Convergence. John Wiley \& Sons, New York, 1986.

[9] G. M. Ewing. Calculus of Variations with Applications. Dover Publications, New York, 1985.

[10] R. P. Feynman. The principle of least action in quantum mechanics. Unpublished doctoral dissertation, Princeton University, Princeton, NJ, 1942.

[11] M. C. Fu, T. G. Marcus, and I. Wang. Monotone optimal policies for a transient queueing staffing problem. Operations Research 48:327-331, 2000.

[12] L. Green and P. Kolesar. The pointwise stationary approximation for queues with nonstationary arrivals. Management Science 37:84-97, 1991.

[13] S. Halfin and W. Whitt. Heavy-traffic limits theorem for queues with many exponential servers. Operations Research 29:567-588, 1981.

[14] R. C. Hampshire. Dynamic queueing models for the operations management of communication services. Unpublished doctoral dissertation, Princeton University, Princeton, NJ, 2007.

[15] R. C. Hampshire and W. A. Massey. Variational optimization for call center staffing. Richard Tapia Celebration of Diversity in Computing, Proceedings of the 2005 Conference on Diversity in Computing, Albuquerque, NM, 2005.

[16] R. C. Hampshire and W. A. Massey. Priority scheduling for a weighted processor sharing queue with time varying rates. Working paper, Carnegie Mellon University, Pittsburgh, 2010.

[17] R. C. Hampshire, M. Harchol-Balter, and W. A. Massey. Fluid and diffusion limits for transient sojourn times of processor sharing queues with time varying rates. Queueing Systems 53:19-30, 2006.

[18] R. C. Hampshire, O. B. Jennings, and W. A. Massey. A time varying call center design with Lagrangian mechanics. Probability in the Engineering and Informational Sciences 23:231-259, 2009 .

[19] R. C. Hampshire, W. A. Massey, and Q. Wang. Dynamic pricing to control loss systems with quality of service targets. Probability in the Engineering and Informational Sciences 23:357-383, 2009.

[20] R. C. Hampshire, W. A. Massey, D. Mitra, and Q. Wang. Provisioning of bandwidth sharing and exchange. Telecommunications Network Design and Economics and Management: Selected Proceedings of the 6th INFORMS Telecommunications Conferences, Kluwer Academic Publishers, Boston/Dordrecht/London, 207-226, 2002.

[21] W. J. Hopp. Technical note - Identifying forecast horizons for non-homogenous Markov decision processes. Operations Research 37:339-343, 1989.

[22] W. J. Hopp, J. C. Bean, and R. L. Smith. A new optimality criterion for nonhomogeneous Markov decision processes. Operations Research 35:875-883, 1987.

[23] R. L. Horne, A. Mandelbaum, and W. A. Massey. Large scale dynamics for multi-server queues. Working paper, Prenceton University, Princeton, NJ, 2010.

[24] D. L. Jagerman. Some properties of the Erlang loss function. Bell System Technical Journal 53:525-551, 1974.

[25] D. L. Jagerman. Nonstationary blocking in telephone traffic. Bell System Technical Journal 625-661, 1975.

[26] M. I. Kamien and N. L. Schwartz. Dynamic Optimization: The Calculus of Variations and Optimal Control in Economics and Management. Elsevier, Amsterdam, 1991.

[27] F. P. Kelly. Reversibility and Stochastic Networks. John Wiley \& Sons, Chichester, UK, 1979.

[28] L. Kleinrock. Queueing systems. Volume I: Queueing Systems. John Wiley \& Sons, 1976.

[29] J. Kolmós, P. Major, and G. Tusnády. An approximation of partial sums of independent RV'-s, and the sample DF. II. Z. Wahrscheinlichkeitstheorie verw. Gebiete 32:111-131, 1975.

[30] C. Lanczos. The Variational Principles of Mechanics, 4th ed. Dover Publications, New York, 1970.

[31] M. Lewis and S. Yoon. Optimal pricing and admission control in a queueing system with periodically varying parameters. Queueing Systems: Theory and Applications 47:177-199, 2004.

[32] A. Mandelbaum and W. A. Massey. Strong approximations for time-dependent queues. Mathematics of Operations Research 20:33-64, 1995.

[33] A. Mandelbaum, W. A. Massey, and M. Reiman. Strong approximations for Markovian service networks. Queueing Systems 30:149-201, 1998.

[34] J. Marsden and T. Ratiu. Introduction to Mechanics and Symmetry. Springer, New York, 1994. 
[35] W. A. Massey. Nonstationary queues. Unpublished doctoral dissertation, Stanford University, Stanford, CA, 1981.

[36] W. A. Massey. Asymptotic analysis of the time dependent $M / M / 1$ queue. Mathematics of Operations Research 10:305-327, 1985.

[37] W. A. Massey and W. Whitt. An analysis of the modified offered load approximation for the nonstationary Erlang loss model. Annals of Applied Probability 4:1145-1160, 1994.

[38] W. A. Massey and W. Whitt. Uniform acceleration expansions for Markov chains with timevarying rates. Annals of Applied Probability 8:1130-1155, 1998.

[39] B. L. Miller. Finite state continuous time Markov decision processes with finite planning horizon. SIAM J. Control 6:266-280, 1968.

[40] P. Pedregal. Introduction to Optimization (Texts in Applied Mathematics). Springer Science + Business Media, New York, 2004.

[41] L. S. Pontryagin, V. G. Boltyanshii, R. V. Gamkredlidze, and E. F. Mishchenko. The Mathematical Theory of Optimal Processes. John Wiley \& Sons, New York, 1962.

[42] A. Prékopa. On Poisson and composed Poisson stochastic set functions. Studia Mathematica 16:142-155, 1957.

[43] M. L. Puterman. Markov Decision Processes: Discrete Stochastic Dynamic Programming. John Wiley \& Sons, Chichester, UK, 1994.

[44] R. Sato and R. V. Ramachandran. Symmetry and Economic Invariance: An Introduction (Research Monographs in Japan-U.S. Business and Economics). Kluwer Academic Publishers, Norwell, MA, 1998.

[45] L. I. Sennott. Stochastic Dynamic Programming and the Control of Queueing Systems. WileyInterscience, New York, 1999.

[46] S. P. Sethi and G. Thompson. Optimal Control Theory: Applications to Management Science and Economics. Springer-Verlag, New York, 2000.

[47] S. Zeltyn and A. Mandelbaum. Call centers with impatient customers: Many-server asymptotics of the $M / M / n+G$ queue. Queueing Systems, Vol. 51. Springer, New York, 2005. 